\title{
RADIATIVE TRANSFER AND STARLESS CORES
}

\author{
Eric Keto, ${ }^{1}$ George B. Rybicki, ${ }^{1}$ Edwin A. Bergin, ${ }^{2}$ and René Plume ${ }^{3}$ \\ Received 2003 July 14; accepted 2004 June 1
}

\begin{abstract}
We develop a method of analyzing radio-frequency spectral line observations to derive data on the temperature, density, velocity, and molecular abundance of the emitting gas. The method incorporates a radiative transfer code with a new technique for handling overlapping hyperfine emission lines within the accelerated $\Lambda$-iteration algorithm and a heuristic search algorithm based on simulated annealing. We apply this method to new observations of $\mathrm{N}_{2} \mathrm{H}^{+}$in three Lynds clouds thought to be starless cores in the first stages of star formation and determine their density structure. A comparison of the gas densities derived from the molecular line emission and the millimeter dust emission suggests that the required dust mass opacity is about $\kappa_{1.3 \mathrm{~mm}}=0.04 \mathrm{~cm}^{2} \mathrm{~g}^{-1}$, consistent with models of dust grains that have opacities enhanced by ice mantles and fluffy aggregrates.
\end{abstract}

Subject headings: ISM: individual (L1489, L1517, L1544) — ISM: molecules — radiative transfer — stars: formation

\section{INTRODUCTION}

In this paper we show how we can use radiative transfer modeling and heuristic search algorithms to derive the physical conditions in molecular clouds, in particular, their temperature, density, and velocity structure, from radio-frequency spectral line observations. We address some difficulties with this approach. First, the amount of data available from spectral line observations is necessarily less than the amount of data required to fully describe the clouds. Whereas the temperature, density, and velocity of the clouds represent 15 dimensions of data, three dimensions each for the temperature and density fields and nine for the velocity vectors, the data, which consist of the spectral line brightnesses as a function of position projected on the plane of the sky and velocity projected on the line of sight, contain only three dimensions of information. Second, the parameters that we seek are not uniquely related to the observed spectral line brightness. For example, the line brightness is related to the gas temperature and the column density, which is itself a function of both the number density and the path length or size of the cloud. Different combinations of temperature, density, and size can produce the same line brightness. Third, even for simple cloud models, the possible combinations of cloud size, density, temperature, and velocity structure are too many to permit an exhaustive search.

Nevertheless, we can make some progress. We can reduce the number of parameters needed to describe the clouds by considering simplified geometries rather than fully threedimensional models. Within the context of these simplified models, some of the variables that are not uniquely determined by the data are much better constrained. For example, in the context of spherically symmetric models, both the number density and the radial size of a cloud can be determined from a map of the cloud. Similarly, observations of multiple spectral lines, particularly hyperfine lines within rotational transitions,

\footnotetext{
${ }^{1}$ Harvard-Smithsonian Center for Astrophysics, 60 Garden Street, Cambridge, MA 02138.

2 Department of Astronomy, University of Michigan, 825 Dennison Building, 501 East University Avenue, Ann Arbor, MI 48109.

3 Department of Physics and Astronomy, University of Calgary, 2500 University Drive NW, Calgary, AB T2N-1N4, Canada.
}

can be used to separate the density and temperature because the ratios of the hyperfine lines are most sensitive to the column density, while the ratios of different rotational transitions are more sensitive to the temperature. Finally, while it is still not feasible to do an exhaustive search through the parameter space of even simplified models, heuristic search algorithms such as simulated annealing (SA) are effective in narrowing the range of parameters and finding models consistent with the data. Furthermore, the history of the search through the available models can be used to estimate the uncertainties in each of the parameters describing the molecular cloud.

To explore these techniques, we have combined a non-LTE, radiative transfer (RT) code using the accelerated $\Lambda$-iteration (ALI) algorithm of Rybicki \& Hummer (1991), with a search code using an adaptation of the fast SA (FSA) algorithm of Szu \& Hartley (1987). The RT code generates synthetic spectral line profiles from parameterized models of molecular clouds, while the FSA code adjusts the parameters of the models to minimize the $\chi^{2}$ difference between observed and synthetic spectra. The parameters, typically defining the gas temperature, number density, and velocity, then represent the physical description of the cloud derived from the data.

For most molecules observed in radio astronomy, the collision rates have been calculated only for the transitions between rotational levels, not for the transitions between hyperfine levels. The hyperfine level populations therefore cannot be determined with the full non-LTE equations. Therefore, our RT code uses the approximation that the hyperfine levels within a single rotational level are populated according to their statistical weights, while the rotational transitions are treated through a more complete non-LTE calculation (Keto 1990). This approach also allows the simpler ALI algorithm for nonoverlapping lines (Rybicki \& Hummer 1991) to be used, even in cases in which the hyperfine lines overlap, rather than the more complex ALI algorithm for overlapping lines (Rybicki \& Hummer 1992). This technique for treating hyperfine lines can be adopted for use with any non-LTE RT code by the rather simple modification of replacing the local spectral line profile, usually a Gaussian, with an equivalent complex profile consisting of the individual hyperfine lines weighted by their relative line strengths. 
We apply these techniques to observations of starless cores in Lynds clouds to determine their structure. The starless cores are thought to be gas clouds in the earliest stages of star formation that are just beginning gravitational collapse to form protostars. Their internal structure and the consequences of that structure for the formation of protostars is an area of active research. In a series of papers, Myers et al. (1983), Myers \& Benson (1983), and Benson \& Myers (1989) identified a number of dense cores in molecular clouds as sites of future star formation. On the basis of IRAS observations, Beichman et al. (1986) separated the cores into two classes, those containing stars and those without, and proposed the starless cores as the earlier evolutionary phase, in which star formation had not yet begun. On the basis of the results of a submillimeter continuum survey of the starless cores, WardThompson et al. (1994) also concluded that the starless cores were in a stage preceding star formation. They found insufficient luminosity for accreting protostars and on the basis of statistical arguments found the cores to be long lived, with timescales consistent with ambipolar diffusion but not with gravitational free fall, which would be associated with protostars. However, in the case of some cores, Tafalla et al. (1998), Lee \& Myers (1999), and Lee et al. (2001) came to different conclusions. Their observations indicated short lifetimes of $\sim 1$ Myr and the presence of inward motions of about $0.1 \mathrm{~km} \mathrm{~s}^{-1}$.

Two different observational surveys derived the density structure of the starless cores. Ward-Thompson et al. (1999) mapped the starless cores in the millimeter continuum dust emission, and Bacmann et al. (2000) mapped the cores in $7 \mu \mathrm{m}$ dust absorption against the infrared background. Both studies arrived at similar conclusions: the density structure of the starless cores is characterized by a core-envelope structure with an inner region of approximately constant or weakly decreasing density and a surrounding envelope with a steep density gradient.

However, Evans et al. (2001) and Shirley et al. (2002) argued that the dust observations cannot be interpreted without knowledge of the dust temperature, which is not available from the observations. Therefore, the observations may not rule out such steep density profiles after all. They and also Zucconi et al. (2001), Stamatellos \& Whitworth (2003), and Goncalves et al. (2004) calculated that the temperature in starless cores should decrease inward. Evans et al. (2001) suggested that the density profiles of Ward-Thompson et al. (1999) that had been derived assuming constant dust temperature may be incorrect, and the actual density profile could be much steeper, for example, as steep as $r^{-2}$. As for the dust absorption observations (Bacmann et al. 2000), they are not affected by variations in the dust temperature and would seem to confirm the earlier results derived from the dust emission. Evans et al. (2001) argued that other errors in method invalidated these results as well.

Different studies came to different conclusions about the value of the gas density. Andre et al. (1996), Ward-Thompson et al. (1999), Evans et al. (2001), and Tafalla et al. (2002, 2004) derived gas densities from observations of dust emission that were typically about an order of magnitude greater than the densities derived from the dust absorption observations of Bacmann et al. (2000).

The structure and mass of the clouds relate to their evolution. Whitworth \& Ward-Thompson (2001) and Alves et al. (2001) suggest that the core-envelope density structure may be indicative of pressure-supported Bonnor-Ebert spheres, while
Ward-Thompson et al. (1994) and Andre et al. (1996) suggest that the structure is consistent with magnetic support and ambipolar diffusion. The flattening of the density profiles in the core centers is at odds with models of protostellar formation that are characterized by rapid collapse, since these models predict steep density gradients approaching the freefall profile with $\rho \sim r^{-3 / 2}$ or $\rho \sim r^{-2}$ (Larson 1969; Larson \& Starrfield 1971; Shu 1977). Finally, the mass of the clouds largely determines their stability to gravitational collapse (Bonnor 1956; Hunter 1977; Whitworth \& Summers 1985; Hasegawa 1988; Foster \& Chevalier 1993).

While the previous observational studies relied heavily on the interpretation of dust emission and absorption to determine the density structure of the starless cores, molecular line observations can also yield information on the density structure and mass, as well as the velocity structure, which also bears on the evolution of the clouds. We observed the starless clouds L1544, L1489-NH3, and L1517B in the $\mathrm{N}_{2} \mathrm{H}^{+}(1-0)$ and (3-2) lines. The molecular line observations have an advantage over the dust continuum observations in that the temperature, density, and velocity structures may be derived self-consistently from the same data set. This self-consistency prevents errors in the analysis of one independent data set from affecting the analysis of another data set, as could be the case if the gasdust temperature is determined theoretically, the gas density is determined from dust absorption observations, and the molecular abundance is based on the dust density.

To be sure, the interpretation of molecular line observations is also difficult, being subject to the complexities of cloud chemistry and spectral line RT. However, in the study of dark clouds the $\mathrm{N}_{2} \mathrm{H}^{+}$molecule offers some advantages that help to minimize the uncertainties due to chemistry and RT. Other molecular tracers, such as $\mathrm{CO}$, are thought to vary in abundance because of freezeout onto grains in the cold interiors of molecular cores (Brown et al. 1988; Willacy \& Williams 1993; Hasegawa et al. 1992; Hasegawa \& Herbst 1993; Caselli et al. 1999, 2002b, 2002c; Bergin et al. 2002; Tafalla et al. 2002). In contrast, both observations and theory suggest that $\mathrm{N}_{2} \mathrm{H}^{+}$ maintains a relatively constant abundance with respect to $\mathrm{H}_{2}$ throughout a starless core. (Bergin et al. 1995, 2002; Aikawa et al. 2001; Caselli et al. 2002a). Second, the hyperfine splitting of the rotational transitions of the $\mathrm{N}_{2} \mathrm{H}^{+}$molecule caused by the electric quadrupole interactions of the nitrogen atoms provides additional information on the opacity of each rotational transition that aids in constraining the excitation analysis. With the information provided by the hyperfine lines, it is sufficient in principle to observe only two rotational transitions of this tracer to separate the effects of the gas temperature and density, as well as to probe the velocity structure within the cores.

\section{RADIATIVE TRANSFER AND MODEL CLOUDS}

\subsection{Separating Correlated Parameters}

An advantage that the molecular line observations have over the dust continuum is that the line observations permit a separation of variables that are otherwise correlated. For example, as discussed in the introduction, the dust temperature and density can be traded one for the other to produce the same dust emission. Therefore, an observation of dust emission is insufficient to define both the temperature and density.

Observations of two rotational transitions of a molecule that has hyperfine structure should in principle be sufficient to determine uniquely both the temperature and density. As an 
illustration of the principles, we consider the simple analytic equations that can be used to derive the temperature and density under the assumption that the cloud is a homogeneous layer in LTE. Besides its use as an illustration, such a calculation provides a means to derive the initial guess necessary for the FSA search algorithm and an approximate check on the eventual solution. We emphasize that for the main calculations of this paper, which derive the parameters of the starless cores, we do not use this simple model but use our full non-LTE RT code.

Our illustrative model assumes the simplest RT through a cloud of optical depth $\tau$ with constant source function $S$ between the upper and lower states of a line. In this case, the intensity of a spectral line relative to the background intensity $I_{\mathrm{bg}}$ is

$$
\Delta I=\left(I-I_{\mathrm{bg}}\right)=\left(S-I_{\mathrm{bg}}\right)\left(1-e^{-\tau}\right) .
$$

This relation may be applied to two hyperfine lines, (1) and (2), of, for example, the (1-0) rotational transition of $\mathrm{N}_{2} \mathrm{H}^{+}$, if we assume that the source function is the same in both lines. The optical depth in one of the hyperfine lines, for instance, $\tau_{1-0}^{(1)}$, can be estimated from the observed ratio,

$$
\frac{\Delta I_{1-0}^{(1)}}{\Delta I_{1-0}^{(2)}}=\frac{1-\exp \left(-\tau_{1-0}^{(1)}\right)}{1-\exp \left(-f \tau_{1-0}^{(1)}\right)},
$$

where $f$ is the ratio of the absorption coefficients in the two hyperfine lines, which is given by hyperfine theory. What is observed is not the line intensity itself, but rather the antenna temperature, which is related to the line intensity through a multiplicative factor, $T^{A}=\beta \Delta I$. This factor depends on the characteristics of the antenna, which can usually be measured, and the filling factor of the cloud within the antenna beam, which is usually unknown. However, because the hyperfine lines are typically quite close to each other in frequency, the beam sizes of the two measurements are quite close, and the multiplicative factors, $\beta$, cancel in the ratio of two lines,

$$
\frac{T_{1}^{A}}{T_{2}^{A}}=\frac{\Delta I_{1}}{\Delta I_{2}} .
$$

Thus, the ratio of the measured antenna temperatures of the two hyperfine lines can be used to determine the optical depth.

The optical depth is related to the column density and the excitation temperature,

$$
\tau_{1-0}=\int \alpha d s=\frac{c^{2} h A_{u l} N_{1}}{8 \pi \nu_{0} \delta \nu k J\left(T_{x}\right)},
$$

where $\alpha$ is the opacity and $J\left(T_{x}\right)=(h \nu / k) / \exp \left(h \nu / k T_{x}-1\right)$. The column densities of two different rotational levels are related to the excitation temperature and the energy difference between the levels,

$$
\frac{N_{1}}{N_{2}}=\frac{g_{1}}{g_{2}} e^{\Delta E_{12} / k T_{x}} .
$$

Thus, from observations of two hyperfine lines of two rotational transitions we can determine both the column density and temperature.

When we consider more complex models and solve the RT equations numerically, the same considerations that allow the separation of the excitation temperature and density still apply, and in principle, we can determine the number density and temperature uniquely from the observations. However, noise in the data and the finite spatial and spectral resolutions mean that there will always be some uncertainty in the estimates of each parameter. There will also usually appear to be some correlation between some parameters, but both the uncertainties and correlations can be reduced by better quality data.

\subsection{Searching for the Best Fit}

Even uncorrelated parameters are not independent in the context of a search through multiple parameters. For example, in fitting for the temperature and density of a cloud, one strategy might be to run through a series of temperatures to find the best fit, and then run through a series of densities to find the best-fit density given the recently found temperature. However, this technique will not work since the fit of density is good only for that one particular temperature and that temperature is good only for the initial assumed density. Nor will the temperature ever budge off its previous fit unless the density is first changed and vice versa.

An exhaustive grid search would find the best fit but would be too slow. In our study, we minimize $\chi^{2}$ as a function of seven model parameters: abundance, temperature, core density and size, the exponent of the power law for the density in the envelope, and the infall velocity or microturbulent line width. If there are seven parameters to fit and the range of each parameter is to be spanned by 10 trials, for example, sampling the temperature range from 8 to $18 \mathrm{~K}$ in $1 \mathrm{~K}$ steps, then the total number of trials would be $10^{7}$. At $100 \mathrm{~s}$ per trial, the time required for the exhaustive grid search would be over 10 years of computing.

Heuristic search algorithms such as SA have been found to be effective in searching multidimensional parameter spaces with reasonable efficiency and accuracy. In our study we use an improved variation of SA called FSA, which converges on a linear rather than a logarithmic timescale.

The SA algorithm operates by randomly guessing new values for the parameters and adopting the new values if the new model has a lower $\chi^{2}$. To reduce the chance that the solution will be trapped in a local minimum of the parameter surface and not find the best-fitting global minimum, on the basis of a probability schedule the algorithm is occasionally allowed to adopt a new parameter value even if the computed $\chi^{2}$ indicates a worse fit. This allows occasional uphill movement and lets the search climb out of local minima. Convergence is controlled by reducing the probability of acceptance of uphill moves over time. Thus, there are two probability distributions in the algorithm: the first is the probability of generating a new parameter some distance from the current value, and the second is the probability of accepting a new model that has a $\chi^{2}$ higher than the current model. The original SA algorithm employed a Gaussian distribution for the first probability distribution and an exponential distribution for the second, $\exp \left(-\chi^{2} / T\right)$, where $T$ is the so-called annealing temperature, which is reduced over time (Metropolis et al. 1953). The analogy with cooling gives the algorithm its name. The FSA algorithm of Szu \& Hartley (1987) employs a Cauchy probability function in place of the Gaussian. The wider wings of the Cauchy distribution allow larger jumps, searching the parameter space faster. This in turn allows the annealing temperature to be reduced more quickly, and thus, the algorithm converges on the global minimum more quickly. We further modify the original Metropolis algorithm to allow 
for a search in a continuous rather than a discrete parameter space. This is done by setting the magnitude of the variation of each parameter to an amount expected to produce a change in $\chi^{2}$ equal to the annealing temperature. As the annealing temperature is reduced over time, the magnitude of the variation of the parameters is also reduced so that the searched volume contracts in parameter space as the algorithm converges. Because the magnitude of variation for each parameter is not known a priori and, furthermore, changes as the parameters of the models change, the variations of each parameter necessary to produce the desired variation of $\chi^{2}$ are estimated from the results of the last several iterations as the computation progresses. A search with SA is certainly not guaranteed to find a global minimum, but in practice the results are generally useful.

How best to estimate the uncertainty in the solution for each parameter or the range of each parameter that is consistent with the data is in practice somewhat of an open question. In theory, the range is given by the curvature of the $\chi^{2}$ surface in multidimensional parameter space. However, this surface is not easy to display once the number of dimensions exceeds two or three. For example, if we suppose that we have found the global minimum of our parameters, then for any particular parameter, the second derivative of $\chi^{2}$ with respect to that parameter, that is, the curvature, can be used as an indication of the uncertainty. If the $\chi^{2}$ surface were relatively flat along the dimension of the parameter, then relatively large variations in the parameter would not appreciably change $\chi^{2}$, the model would not be sensitive to that parameter, and the constrained range would be relatively large. However, even if the surface is steeply curved along the direction of one parameter, indicating a narrow range and high precision, we cannot know from this analysis if the parameter is correlated with other parameters so that it would in fact have a large range if one or more of the other parameters were allowed to change. For example, in the case of the dark clouds the abundance of $\mathrm{N}_{2} \mathrm{H}^{+}$ with respect to $\mathrm{H}_{2}$ and the density of $\mathrm{H}_{2}$ itself can be traded one for the other to some extent. However, if we fixed the abundance, the density would appear to be very tightly constrained indeed. If we had only two parameters to deal with, we could easily plot the $\chi^{2}$ surface, for example, as contours, to understand the correlation. However, with additional parameters, for example, the cloud size, one can be traded to some extent for the number density, as well as the abundance, to produce equivalent column densities, and it is difficult in practice to display and understand the correlations.

The collection of models calculated by the SA algorithm can be organized to provide estimates of the uncertainties on each of the fitted parameters. If we project the parameters of all the multidimensional models onto each dimension one at a time, the projection results in a one-dimensional surface (a line) formed by the minima of the multidimensional $\chi^{2}$ surface in projection. The curvature of that line describes the range of each parameter, allowing for, or regardless of, any variation in the other parameters that could result in a plausible model. The apparent ranges are overly conservative, too broad, because much of the variation involves correlations with parameters that may have values far from their optima. Nevertheless, a plot of projected $\chi^{2}$ is visually obvious, and the ranges found are narrow enough to be interesting with respect to our understanding of conditions in the starless cores.

A second method of displaying uncertainties that is particularly helpful in examining correlations among the fitted parameters is to plot the $\chi^{2}$ surface as a function of two parameters at a time. Correlations, for example, between abundance and density, would show up as a line of lower $\chi^{2}$ across the surface at an orientation that is not parallel to either surface. Parameters that are not correlated have a $\chi^{2}$ minimum that is a single point on the surface or a line parallel to a surface. The latter indicates a larger range in one parameter than in the other. Of course, there may be correlations among three or more variables in the analysis, but it is difficult to display more than two at a time. Examples of these plots are presented in the sections describing the analyses of the clouds.

\subsection{ALI for Molecules with Hyperfine Lines}

For the numerical simulation, we developed a code that employs the accelerated $\Lambda$-iteration algorithm of Rybicki \& Hummer (1991) and solves the RT equation in a threedimensional grid. While many such codes employ the method of short characteristics (Kunasz \& Auer 1988) to solve the RT equation, we use a method based on long characteristics. In our method the rays, distributed in angle and impact parameter, each pass through the entire volume of the model. The intensity field at each spatial point is found by simple integration along each of these rays. The various radiation quantities required by the method are found by appropriate frequency and angle averaging over the rays that pass through each cell or voxel in the model cube. Since the brightness along each ray is calculated only twice, once in the forward and once in the reverse direction, the computational burden of this method of long characteristics is quite similar to that of short characteristics. Some numerical tests of our code are described in the Appendix.

The averaging of the line radiation over frequency that produces the local mean radiation field, $J$, requires some explanation for molecules such as $\mathrm{N}_{2} \mathrm{H}^{+}$that show hyperfine splitting of the rotational levels, with the hyperfine lines overlapping within the width of the line profile. Such overlapping transitions can, in principle, be treated with the extended formalism for the accelerated $\Lambda$-iteration algorithm of Rybicki \& Hummer (1992), but this requires that the collision rates between individual hyperfine states be known. Typically, for radio-frequency molecular rotational transitions, the collision rates are known only for the total of all the hyperfine transitions between rotational levels. However, it would be inaccurate to solve the RT equation while ignoring the hyperfine splitting, because this would result in a single line with an unrealistic optical depth and, in cases of moderate to high optical depth, a saturated line with an inaccurate brightness and width.

We use here an approximate treatment of the hyperfine transitions in which the hyperfine levels within each rotational level are assumed to be populated in proportion to their statistical weights. Denoting a general rotational level by the Roman suffix $i$ and the associated hyperfine sublevel by the Greek suffix $\alpha$, this assumption may be expressed as

$$
n_{i \alpha}=\frac{g_{i \alpha}}{g_{i}} n_{i}
$$

where the $n$-values are the populations and the $g$-values are the statistical weights. This assumption allows us to reformulate the statistical equilibrium equations so that only the total rotational populations $n_{i}$ appear. The collisional and radiative rate coefficients between these rotational levels are given by averages over the hyperfine transitions with the usual weighting by statistical weights. 


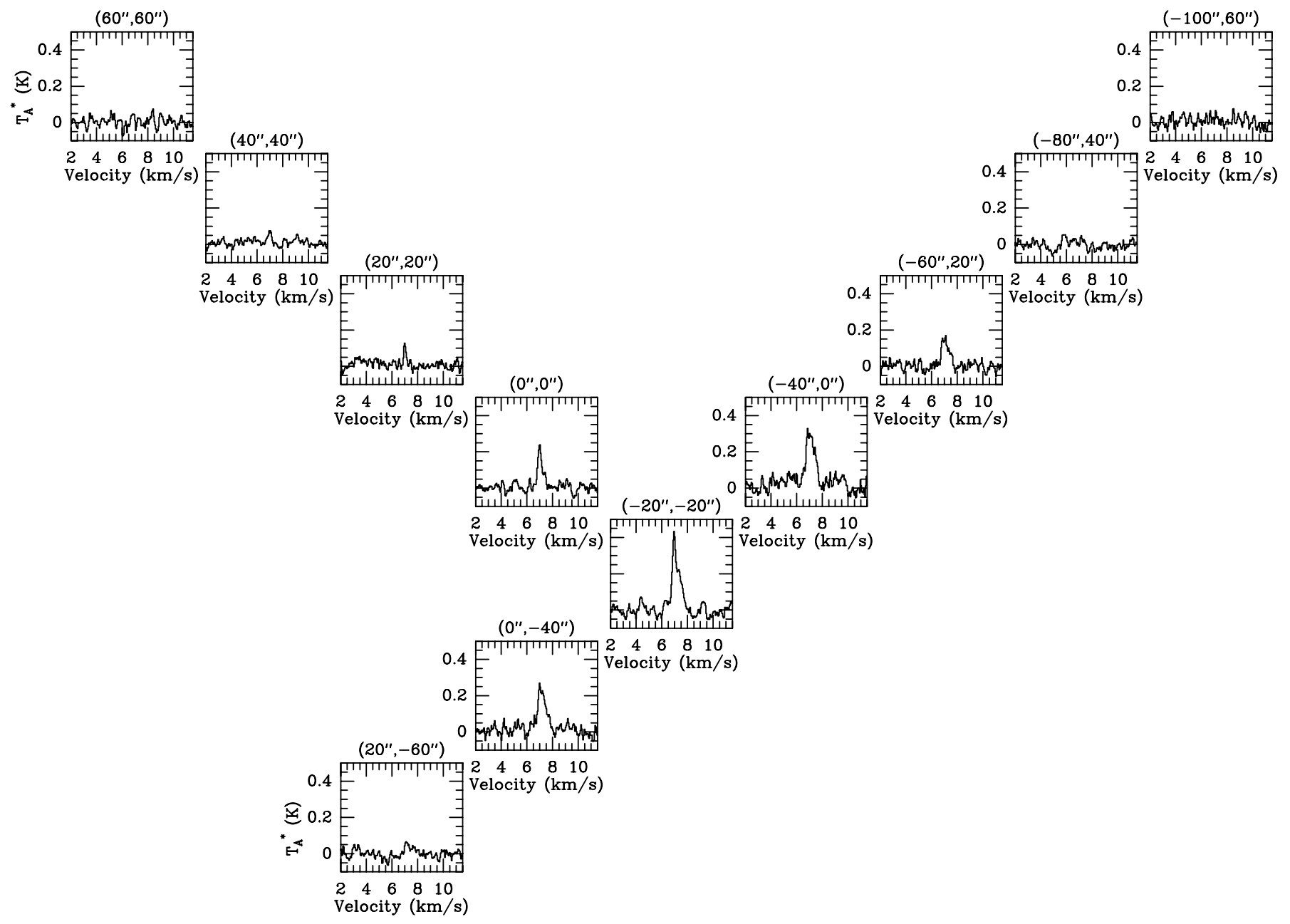

FIG. 1. $-\mathrm{N}_{2} \mathrm{H}^{+}(3-2)$ spectra at observed positions in L1544. The spectra are uncorrected for antenna loss.

In particular, the radiative rates between the rotational levels $i$ and $j$ are properly taken into account by substituting for the Gaussian line profile function of a single unsplit line the more complex line profile function

$$
\phi_{i j}(\nu)=\sum_{\alpha \beta} R_{i \alpha, j \beta} \phi_{i \alpha, j \beta}(\nu),
$$

that is, the sum of the Gaussian profiles $\phi_{i \alpha, j \beta}$ of each of the individual hyperfine transitions from $i \alpha$ to $j \beta$, each weighted by the individual relative line strength $R_{i \alpha, j \beta}$ and each with a common line width that could include both thermal and microturbulent components, as well as a velocity shift due to systematic motions. This approximation has been previously used in LTE RT modeling (Keto 1990) but is also useful in non-LTE modeling. The summed profile can then be used to compute the radiation quantities of the ALI algorithm using the simpler algorithm for nonoverlapping lines, even though the RT equation is solved allowing for the overlapping of the hyperfine lines. This technique can easily be incorporated into any ALI or Monte Carlo code by replacing the line profile function of a single line with the line profile function representing the sum of all the hyperfine components.

The approximation of summing the relative intensities of the hyperfine lines also yields a saving in computational time for molecules such as $\mathrm{N}_{2} \mathrm{H}^{+}$that have overlapping hyperfine lines. This is because the computational burden is proportional to the frequency bandwidth, and the single-profile function of the sum of overlapping lines covers less bandwidth than is covered by the sum of the bandwidths of the individual hyperfine lines.

The assumption of a statistical population of the hyperfine levels might seem to be a good approximation for molecules emitting in the millimeter radio spectrum, since the energy differences of the hyperfine levels are typically in the millikelvin range, whereas the energy differences between rotational levels are several tens to hundreds of kelvins. However, the $\mathrm{N}_{2} \mathrm{H}^{+}(1-0)$ spectra of dark clouds typically show so-called hyperfine anomalies in which hyperfine lines of the same relative strengths nevertheless are observed to have different intensities (Caselli et al. 1995). These anomalies cannot be modeled by the method outlined here, and with the lack of collision rates between hyperfine levels, it is not clear how to improve the method.

The $\mathrm{N}_{2} \mathrm{H}^{+}$line has a particularly complex set of hyperfine components caused by the interaction of the two nitrogen atoms in the molecule. It is not possible to determine by simple analytic formulas the frequencies and relative intensities of the hyperfine components, as is the case with molecules containing a single nitrogen atom such as $\mathrm{NH}_{3}$ (Townes \& Schawlow 1955). The hyperfine ratios and relative strengths of all the rotational levels up to $\mathrm{N}_{2} \mathrm{H}^{+}(10-9)$ were calculated for us by L. Dore of the University of Bologna using a numerical code following Pickett (1991). For the collision rates, 


\section{L1489}
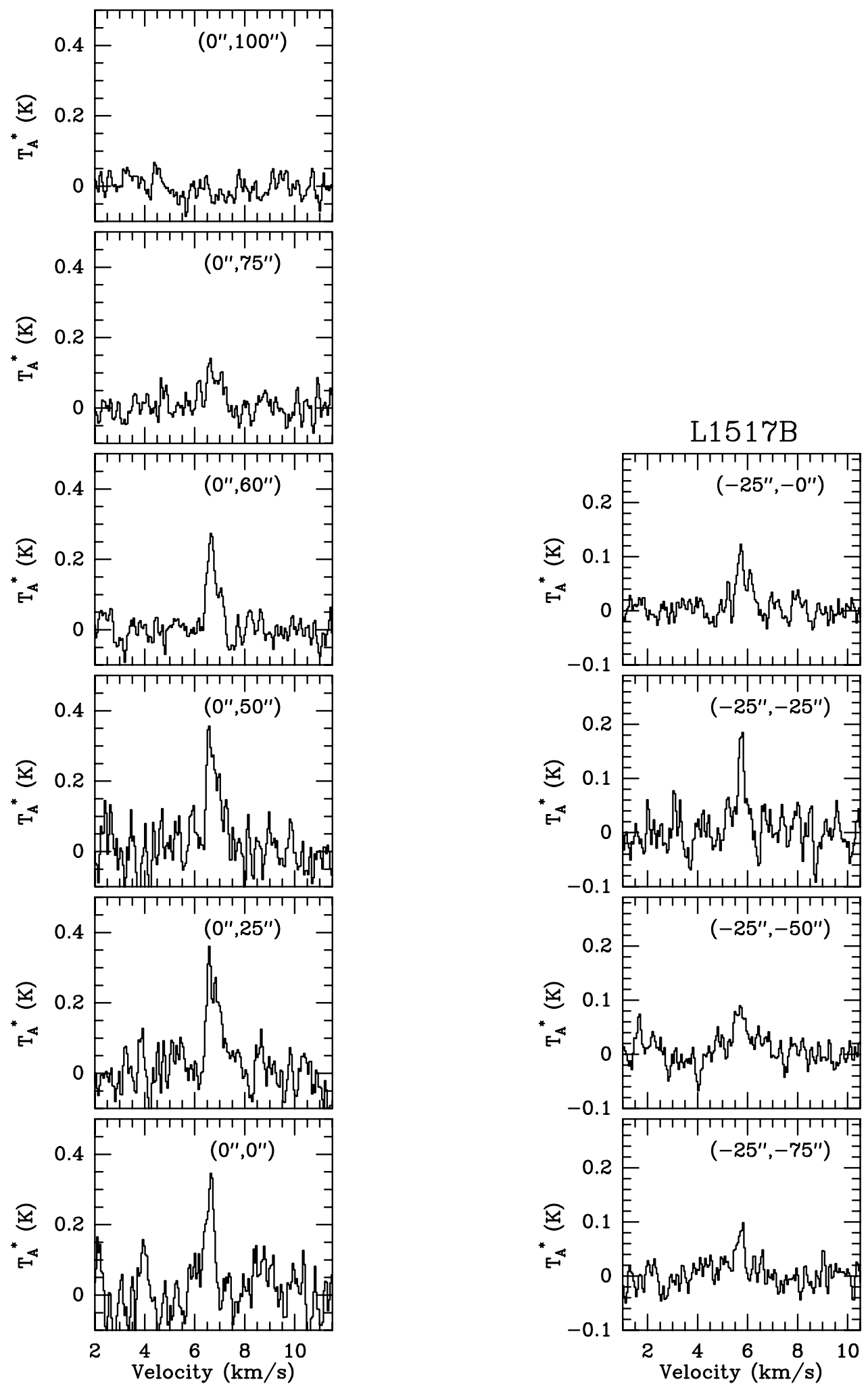

FIG. 2. $-\mathrm{N}_{2} \mathrm{H}^{+}(3-2)$ spectra at observed positions in L1498 and L1517B. The spectra are uncorrected for antenna loss. 

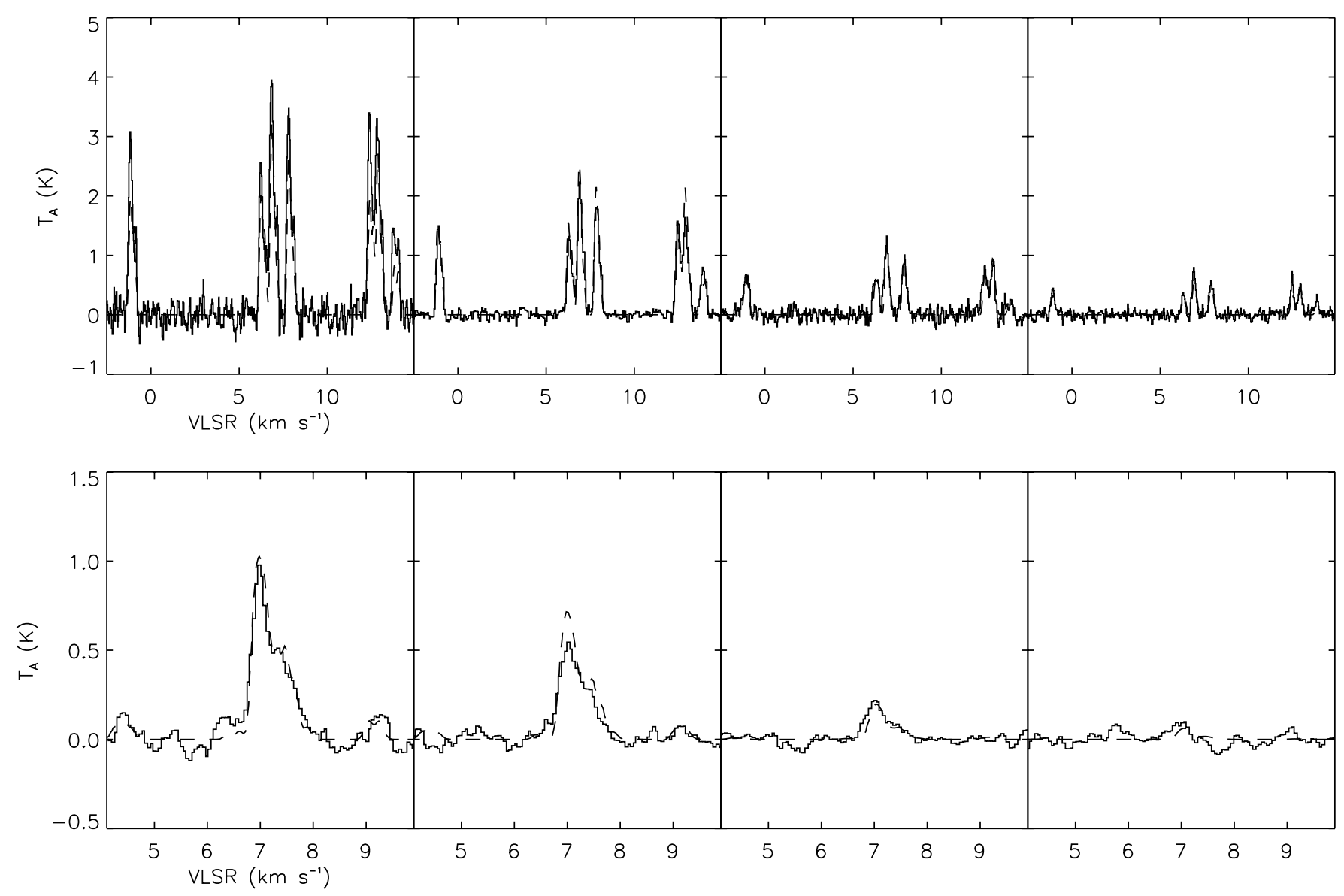

FIg. 3.- Spectra (solid line) and model (dashed line) for L1544. The top row of spectra present the three center hyperfine lines of $\mathrm{N}_{2} \mathrm{H}^{+}(1-0)$ at four positions across the cloud. The spectrum at the left is nearest the center of the cloud (as defined by the dust continuum peak), while the three spectra to the right are from azimuthal averages of the observed spectra, with a separation between radial positions of $20^{\prime \prime}$. The bottom row of spectra shows the central hyperfine components of the $\mathrm{N}_{2} \mathrm{H}^{+}(3-2)$ line from the same positions as the spectra in the first row. The temperature scale of the data has been corrected for antenna loss and atmospheric absorption.

we used the published rates of Flower (1999) calculated for the molecular ion $\mathrm{HCO}^{+}$and presumed to be similar to rates for the molecular ion $\mathrm{N}_{2} \mathrm{H}^{+}$.

\section{PARAMETERIZED MODELS OF THE STARLESS CORES}

We describe the starless cores by simple parameterized models based on previous observations. The density structure of the starless cores is inferred from the dust continuum observations as a constant-density core surrounded by an envelope with a steep density gradient (Ward-Thompson et al. 1994; Bacmann et al. 2000). A simple model for this density structure can be described by three parameters in spherical symmetry: the radius of the central region of constant density, $r_{\text {cor }}$, the density in this region, $n_{\text {cor }}$, and the exponent, $\alpha$, of the power law for the density decrease in the surrounding envelope. While simple, this model is sufficient to describe clouds with density profiles ranging from constant density to continuous power-law profiles with a singularity at the origin.

The temperature structure is suggested by previous theoretical models indicating cooler temperatures in the center of the core (Evans et al. 2001; Zucconi et al. 2001; Shirley et al. 2002; Stamatellos \& Whitworth 2003; Goncalves et al. 2004). We initially allowed our models to have two different temperatures, a constant temperature characterizing the region of the constant-density core, $T_{\text {cor }}$, and a second, higher temperature in the envelope, $T_{\mathrm{env}}$. However, we found that our data were unable to discriminate between models that had different temperatures in the core and envelope and those that had a single constant temperature, and we adopted the simpler constant-temperature model for all the analyses reported in this paper.

Two cores in our study, L1489-NH3 and L1517B, have Gaussian line shapes consistent with a static field with a constant microturbulent broadening added to the thermal broadening. The Gaussian profiles can also be produced by radial infall with velocities of about the same magnitude as the broadening. In L1489-NH3 and L1517B, velocities that are equal to the free-fall velocity of the enclosed mass have about the correct magnitude, and the resulting velocity field, which decelerates inward, does not produce a split line. At the spatial resolution of the data, the effect is simply to broaden the line, resulting in a spectral shape that does not deviate significantly from a Gaussian. Thus, the data for L1489-NH3 and L1517B are consistent with models of either turbulent support, free-fall velocities, or possibly a variety of other weak velocity fields.

One core in our study, L1544, may have an inward velocity field (Tafalla et al. 1998; Williams et al. 1999; Caselli et al. 2002c). For L1544, we tried models with three different velocity fields: (1) a constant radial inward velocity, (2) an infall 


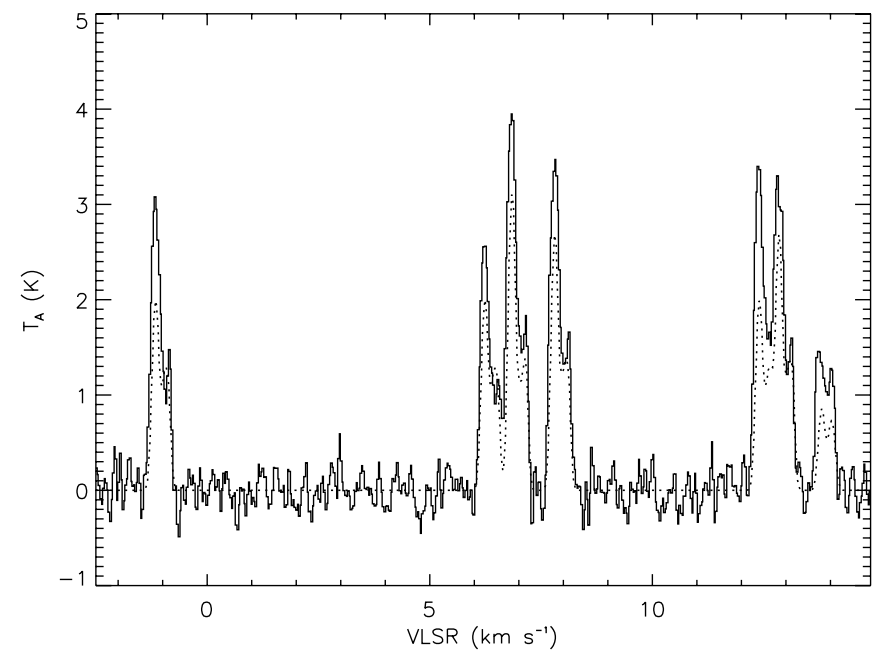

FIG. 4.-First panel of Fig. 3 (top) $\left(\mathrm{N}_{2} \mathrm{H}^{+}\right.$[1-0]; central position) in detail.

velocity equal to the free-fall velocity $v=(G M / r)^{1 / 2}$ of the enclosed mass, $M$, at each radius, $r$, and (3) inward velocities linearly increasing and decreasing as a function of radius. Models with the velocity field varying as the free-fall velocity of the enclosed mass have infall velocities that are higher in the outer parts of the cloud and are therefore unable to reproduce the line splitting because of the self-absorption that is seen in the $\mathrm{N}_{2} \mathrm{H}^{+}(1-0)$ data. Models with a velocity field in free fall toward a point source, such as a protostar, have inwardly accelerating velocities that easily produce the line splitting. However, the high velocities near the point source create wings on the modeled spectra that are not seen in the data. Models with a constant infall velocity are found to provide better fits with some splitting and no strong wings. It is possible to arrange a point source and the gas density distribution to produce an approximately constant velocity field. A better fit is provided by models with velocities that increase linearly from the edge of the cloud to some maximum velocity at the cloud center. These accelerating velocities produce more pronounced splitting than the constant-velocity case but without the line wings from high-velocity gas that are found in the free-fall models. In all these models, the velocity fields are purely diagnostic in that they do not necessarily have any hydrodynamic justification, but any combination of density and velocity is possible if the dynamics is time-variable.

The possible variation of the abundance of $\mathrm{N}_{2} \mathrm{H}^{+}$in the starless cores is an area of active research (Bergin et al. 1995, 2002; Aikawa et al. 2001; Caselli et al. 2002a; Tafalla et al. 2002). In our models we assume that the $\mathrm{N}_{2} \mathrm{H}^{+}$abundance $X_{\mathrm{N}_{2} \mathrm{H}^{+}}$is constant throughout the core and envelope.

TABLE 1

Model Parameters for L1544

\begin{tabular}{|c|c|c|c|}
\hline Parameter & Best Fit & Minimum & Maximum \\
\hline$T(\mathrm{~K}) \ldots \ldots \ldots \ldots$ & 11 & 7 & 14 \\
\hline $\log n_{\text {cor }}\left(\mathrm{cm}^{-3}\right) \ldots \ldots \ldots \ldots \ldots \ldots$ & 5.7 & 5.3 & 6.0 \\
\hline$r_{\mathrm{cor}}(\mathrm{pc})$ & 0.004 & 0.002 & 0.015 \\
\hline$\alpha$ & -1.5 & -2.4 & -1.25 \\
\hline $\log X_{\mathrm{N}_{2} \mathrm{H}^{+}} \ldots \ldots \ldots \ldots \ldots \ldots \ldots \ldots$ & -8.9 & -9.2 & -8.5 \\
\hline$v(r)\left(\mathrm{km} \mathrm{s}^{-1}\right)$ & -0.24 & -0.30 & -0.15 \\
\hline$\Delta v_{\text {turb }}^{a}\left(\mathrm{~km} \mathrm{~s}^{-1}\right) \ldots \ldots \ldots \ldots \ldots \ldots$ & 0.0 & $\ldots$ & $\cdots$ \\
\hline
\end{tabular}

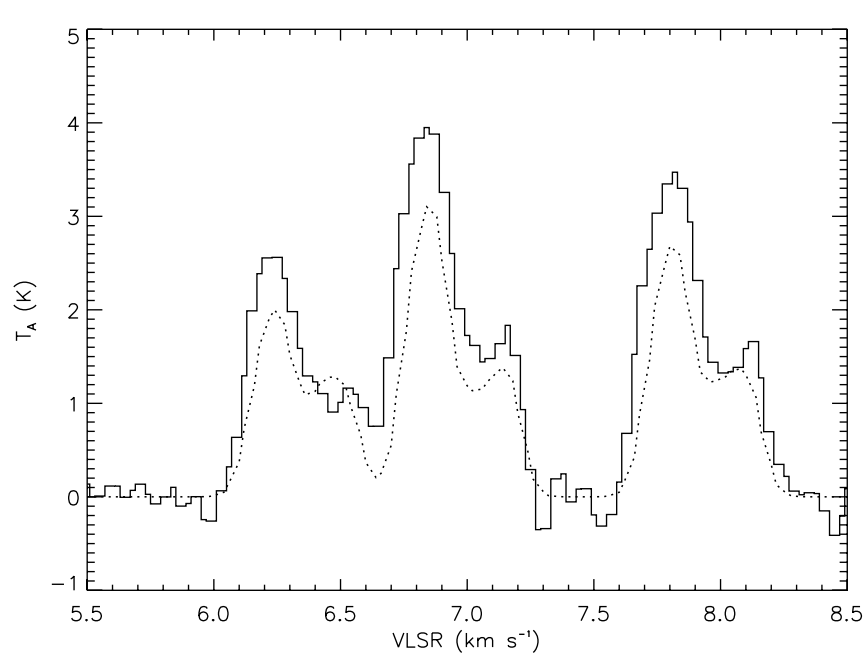

FIG. 5.-First panel of Fig. 3 (top) $\left(\mathrm{N}_{2} \mathrm{H}^{+}[1-0]\right.$; central position) in detail.

The modeling reduces the number of parameters in the description of the clouds to a total of seven. In the constantdensity core we have the core radius and the density. In the surrounding envelope we have the exponent of the density gradient. Finally, for the cloud as a whole we have the temperature, the abundance, the microturbulent line width, and the infall velocity. Although our RT code is three-dimensional, we chose not to add additional parameters to describe the ellipticity of the clouds because, although none of the observed clouds appear truly spherical in the dust continuum, modeling the clouds as ellipses increases the complexity but does not improve our understanding given the limits in our observational data. For two of our clouds we have $\mathrm{N}_{2} \mathrm{H}^{+}(3-2)$ data along only a single axis.

The spherical models were generated within a volume of $0.2^{3} \mathrm{pc}^{3}$ divided into a grid of $20^{3}$ or $32^{3}$ cells. The spectra were computed with a frequency spacing of $0.01 \mathrm{~km} \mathrm{~s}^{-1}$ that was Hanning-smoothed to a final resolution of $0.02 \mathrm{~km} \mathrm{~s}^{-1}$. The radiation field from the model was convolved with a Gaussian of $30^{\prime \prime}$ to represent the effect of the telescope beam, assuming a distance to the clouds of $150 \mathrm{pc}$.

The data are compared with the modeled spectra by computing $\chi^{2}$ over both the $\mathrm{N}_{2} \mathrm{H}^{+}(1-0)$ and $\mathrm{N}_{2} \mathrm{H}^{+}(3-2)$ lines at four radial positions in L1544 that are azimuthal averages of the spectra in Figure 1, four positions in L1489-NH3, and three positions in L1517B.

\section{OBSERVATIONS}

Observations of the $J=3-2$ transition of $\mathrm{N}_{2} \mathrm{H}^{+}(\nu=$ 279.5117 GHz) were obtained using the Caltech Submillimeter Observatory (CSO) $10.4 \mathrm{~m}$ antenna on 1998 February 10-13. Strip maps were completed for three sources, L1544 $\left(\alpha=05^{\mathrm{h}} 04^{\mathrm{m}} 18^{\mathrm{s}} 1, \delta=25^{\circ} 11^{\prime} 08^{\prime \prime}[\mathrm{J} 2000.0]\right)$, L1489-NH3 ( $\alpha=$ $\left.04^{\mathrm{h}} 04^{\mathrm{m}} 47.5, \delta=26^{\circ} 18^{\prime} 42^{\prime \prime} \quad[\mathrm{J} 2000.0]\right)$, and L1517B $(\alpha=$ $04^{\mathrm{h}} 55^{\mathrm{m}} 18.8, \delta=30^{\circ} 38^{\prime} 04^{\prime \prime}$ [J2000.0]). In L1544 the map positions are offset from the continuum peak, which is located at $\Delta \alpha=-20^{\prime \prime}, \Delta \delta=-20^{\prime \prime}$ from our center (Tafalla et al. 2002). The coordinates of $\mathrm{L} 1489-\mathrm{NH} 3$ refer to the $\mathrm{NH}_{3}$ peak in Benson \& Myers (1989) and are different from the coordinates of the nearby IRAS source. To distinguish the molecular cloud from the IRAS source, we refer to the molecular cloud as L1489- $\mathrm{NH}_{3}$. The millimeter dust continuum center in L1489NH3 (Motte \& Andre 2001) is found 37" north of the center of our strip. The millimeter dust continuum peak in L1517B 


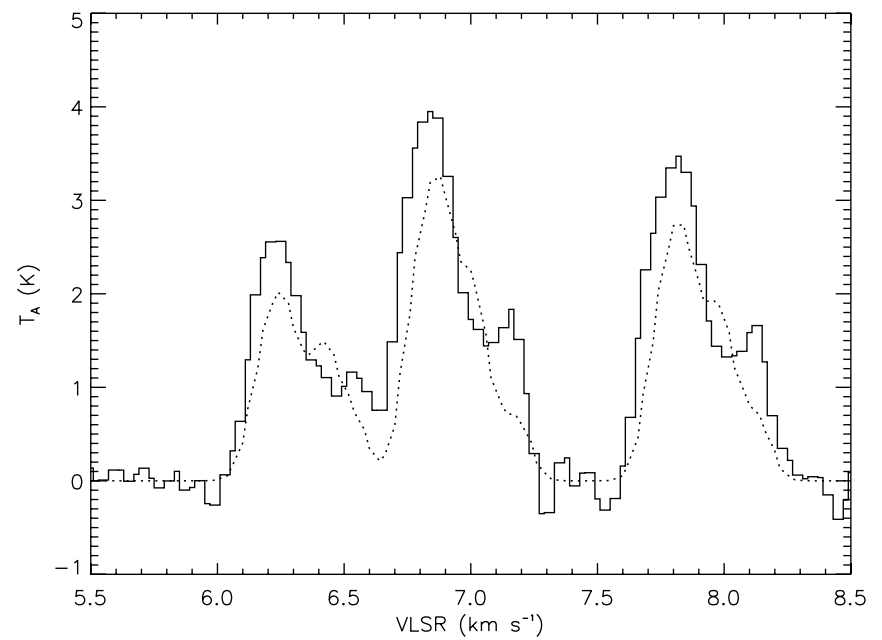

FIG. 6.- Same as Fig. 5, but from a model with a constant infall velocity rather than a linearly accelerating one.

(Tafalla et al. 2002) is offset from the center of our strip by $\left(-20^{\prime \prime},-20^{\prime \prime}\right)$.

The angular and spectral resolutions of these observations were $\sim 26^{\prime \prime}$ and $0.05 \mathrm{~km} \mathrm{~s}^{-1}$, respectively. Pointing during the observations was monitored using Saturn, with a typical rms of less than $2^{\prime \prime}$. Typical system temperatures were $250-$ $350 \mathrm{~K}$. The observation of Saturn produced a double sideband continuum antenna temperature of $T_{A}^{*}=30.5 \mathrm{~K}$. Assuming a source brightness temperature of $135 \mathrm{~K}$ (Hildebrand et al. 1985) and an angular size of 16."8 on 1998 February 12, we estimate a main-beam efficiency of $\eta_{\mathrm{MB}}=0.45$ for our observations.

Our observations of $\mathrm{N}_{2} \mathrm{H}^{+}(1-0)$ in L1517B were obtained with the IRAM 30 m antenna on 1997 August 20. This telescope has a beam size of $\sim 25^{\prime \prime}$ at $93.173 \mathrm{GHz}$, comparable in angular resolution to the CSO beam. The efficiency of the IRAM observations was measured at $B_{\text {eff }}=0.80$, and the spectral resolution was $0.13 \mathrm{~km} \mathrm{~s}^{-1}$.

In our analysis we combined our data with previously published data of $\mathrm{N}_{2} \mathrm{H}^{+}(1-0)$ in $\mathrm{L} 1544$ and L1489-NH3 from Caselli et al. (2002b, 2002c). Caselli et al. observed L1544 using the IRAM $30 \mathrm{~m}\left(\theta_{\mathrm{MB}} \sim 25^{\prime \prime}\right)$ and L1489-NH3 using the FCRAO $14 \mathrm{~m}\left(\theta_{\mathrm{MB}} \sim 56^{\prime \prime}\right)$.

Figures 1 and 2 present the observed $\mathrm{N}_{2} \mathrm{H}^{+}(3-2)$ spectra in L1544, L1489-NH3, and L1517B. In L1544 the positions are along the major and minor axis of the elliptical core as seen in the maps of $\mathrm{N}_{2} \mathrm{H}^{+}(1-0)$ emission in Williams et al. (1999). In all three clouds the strongest $\mathrm{N}_{2} \mathrm{H}^{+}(3-2)$ emission coincides with the continuum peak, and in each case we consistently see a decrease in the emission away from the dust continuum emission peak. In the $\mathrm{N}_{2} \mathrm{H}^{+}(3-2)$ spectra, the red shoulder in
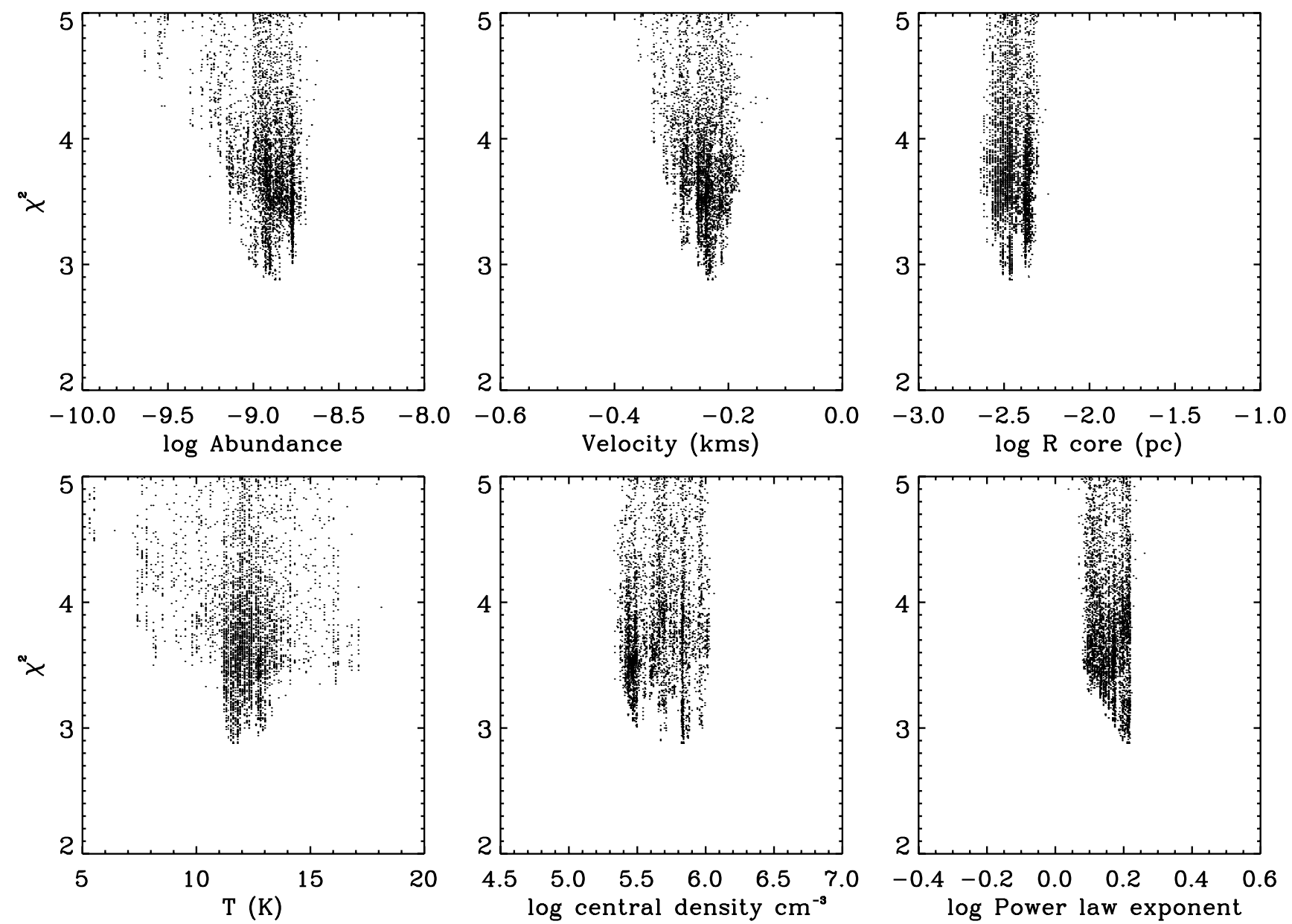

FIG. 7.-Projection of the $\chi^{2}$ surface on the axes of the model parameters for L1544. The lower limit of the points represents an estimate of the curvature of the $\chi^{2}$

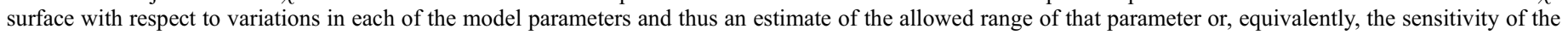
model to variations in that parameter. 

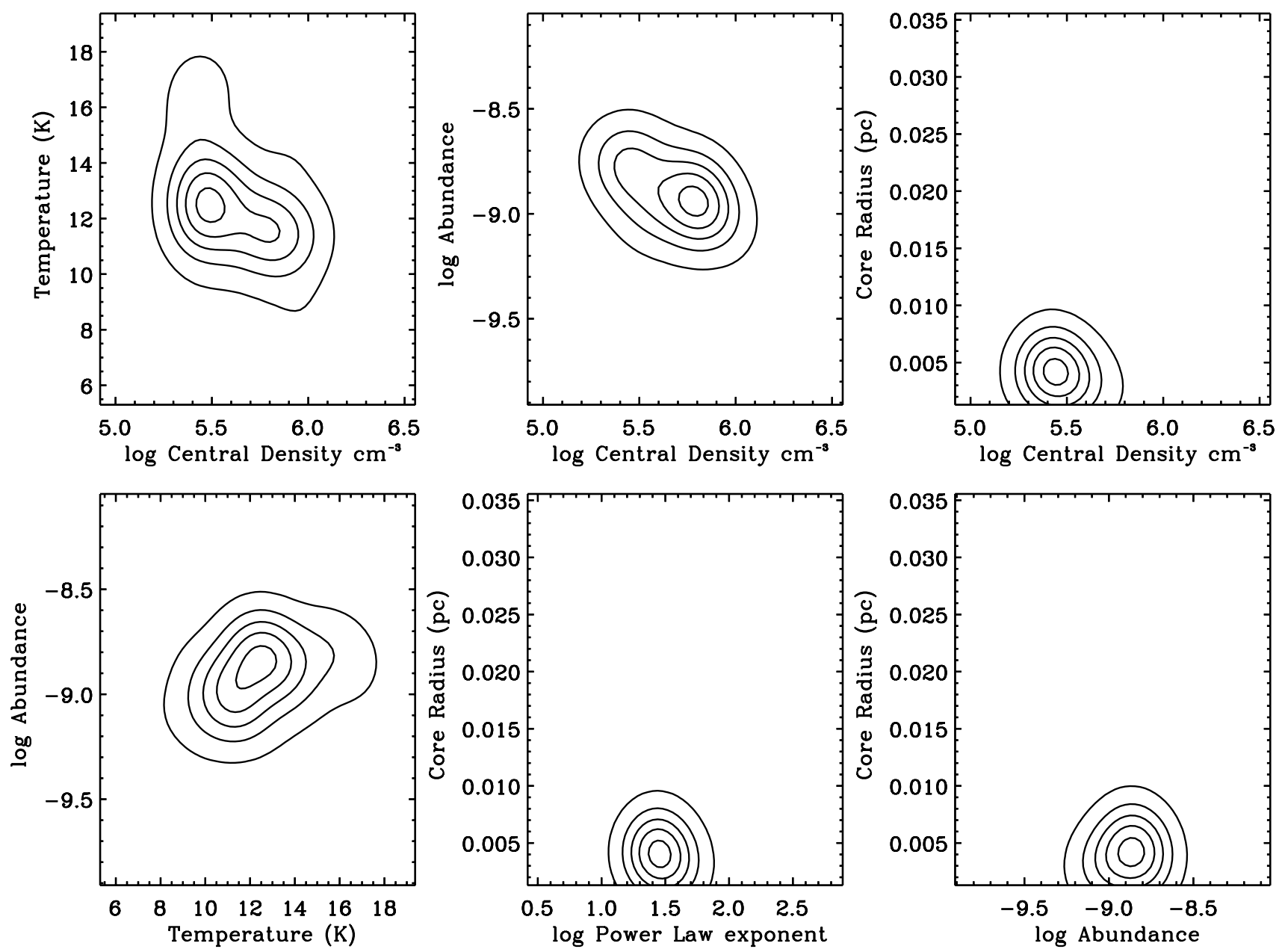

FIG. 8. $-\chi^{2}$ surface as a function of some of the L1544 model parameters taken two at a time. The allowed range for the parameters is indicated by the curvature of the surface. Correlations between parameters would be visible as long valleys with an orientation that is not parallel to either axis. The only significant correlation is between the central density and the abundance. The ranges shown here have a circular shape, indicating that there are no significant correlations between the model parameters. The contour values of $\chi^{2}$ are $3.0-4.0$ in steps of 0.2 .

the emission profile is the result of the blended hyperfine structure of the $J=3-2$ transition rather than self-absorption.

\section{ANALYSIS: INDIVIDUAL CLOUDS}

\subsection{4}

\subsubsection{Best-Fit Model}

A comparison of the data and the spectra for the best-fit accelerating-infall model from a total of several thousand trials is shown in Figures 3 and 4. The model parameters are summarized in Table 1. Figures 5 and 6 show the inner three hyperfine lines of the $(1-0)$ transition for the best-fit linearly accelerating infall model and for comparison a best-fit spectrum from a constant-velocity model. The comparison suggests that the gas in L1544 is not only infalling but also accelerating inward. The absence of line wings on the observed spectra requires that the maximum infall speed be less than $0.2 \mathrm{~km} \mathrm{~s}^{-1}$.

Figure 7 shows the $\chi^{2}$ values of all the models calculated in the search projected onto each of the fitted parameters. As described earlier, the curvature of an imaginary line through the lowest points suggests the range of acceptable values for each parameter. And at each value of a parameter, all the points in a vertical line at the value represent the $\chi^{2}$ of all the models calculated with that value. For example, there are hundreds of different models calculated with a temperature of
$10 \mathrm{~K}$ but with many different variations of all the other parameters. The lowest $\chi^{2}$ at $10 \mathrm{~K}$ represents the best model achieved with that temperature, allowing for any and all variations of the other parameters.

Some of the parameters are more tightly constrained than others. All models with densities below $2 \times 10^{5} \mathrm{~cm}^{-3}$ and above $10^{6} \mathrm{~cm}^{-3}$ result in significantly worse fits to the data. The range of acceptable abundances is between $6 \times 10^{-10}$ and $3 \times 10^{-9}$. The gas temperature cannot be much colder than $7 \mathrm{~K}$, since models with low temperatures do not provide good matches to the spectra, even if the density is increased in the core. The $\chi^{2}$ surface projected onto the axis of the core radius is sparsely populated below 0.002 pc both because core radii below $0.002 \mathrm{pc}$ do not fit the data well and because the volume of the phase space below $0.002 \mathrm{pc}$ is small compared with the volume above $0.002 \mathrm{pc}$. (Phase space volumes can be adjusted mathematically. For example, we fitted the logarithm of the density and abundance to allow a relatively greater volume in phase space at lower parameter values.) The density power law in the envelope must be steeper than $r^{-1.2}$, but the significantly worse fits for core radii below 0.002 pc imply that the density cannot increase as a power law to very small radii.

Possible correlations among the parameters are explored by plotting the surface of $\chi^{2}$ as a function of two parameters at 

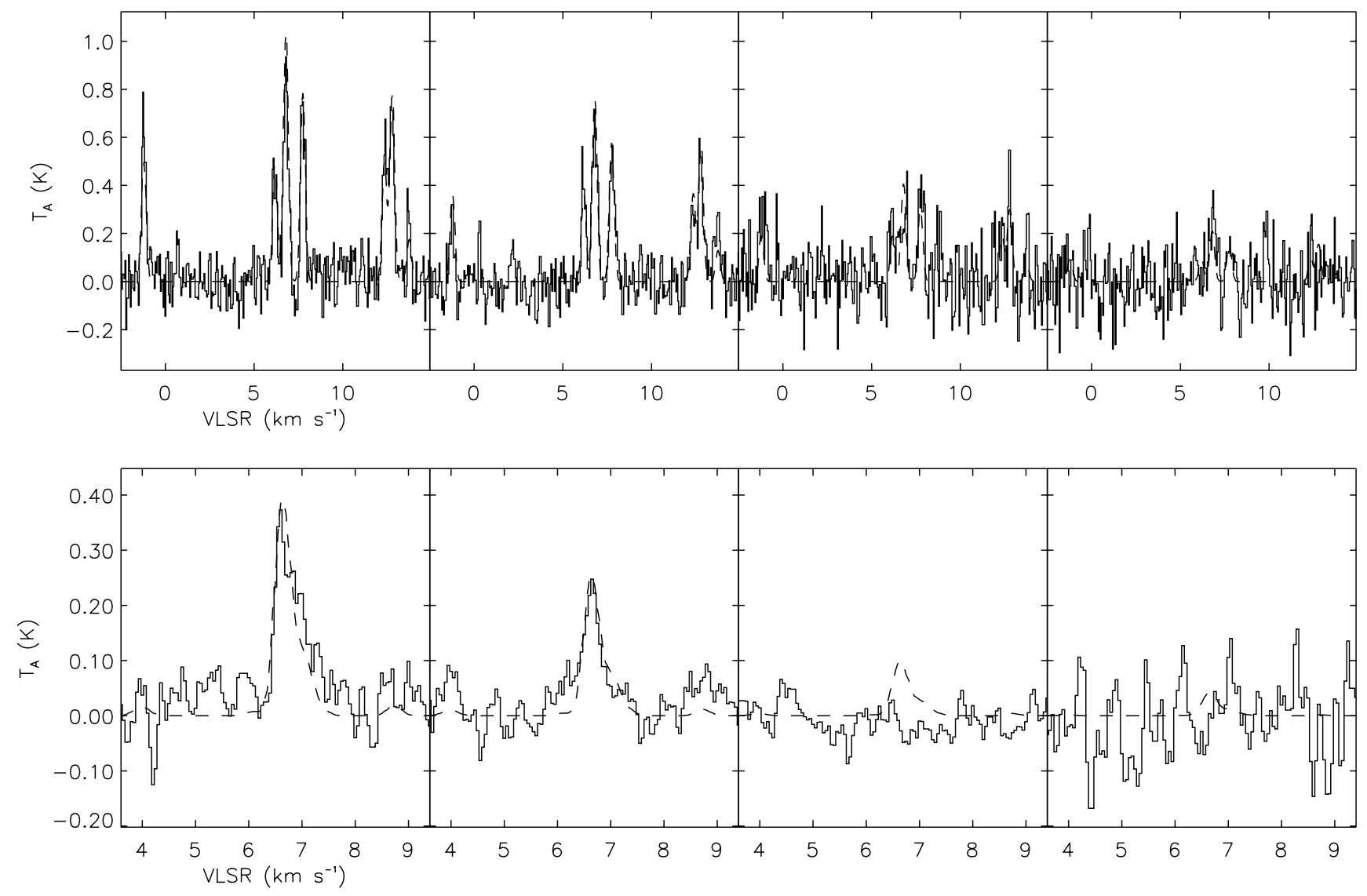

Fig. 9.- Spectra (solid line) and model (dashed line) for L1489-NH3, in the same format as Fig. 3.

a time. Figure 8 shows that there are no strong correlations among the fitted parameters.

\subsubsection{Comparison with Other Results}

Both Ward-Thompson et al. (1999) and Bacmann et al. (2000) derived a density structure from dust observations of L1544 that consists of a constant-density core and a variabledensity envelope, although the latter proposed a steeper density gradient in the envelope. This difference appears reconcilable. The Bacmann et al. (2000) results were derived from observations that cut across the short axis of the elliptical cloud, whereas the Ward-Thompson et al. (1999) results were derived from annular averages of a grid of data.

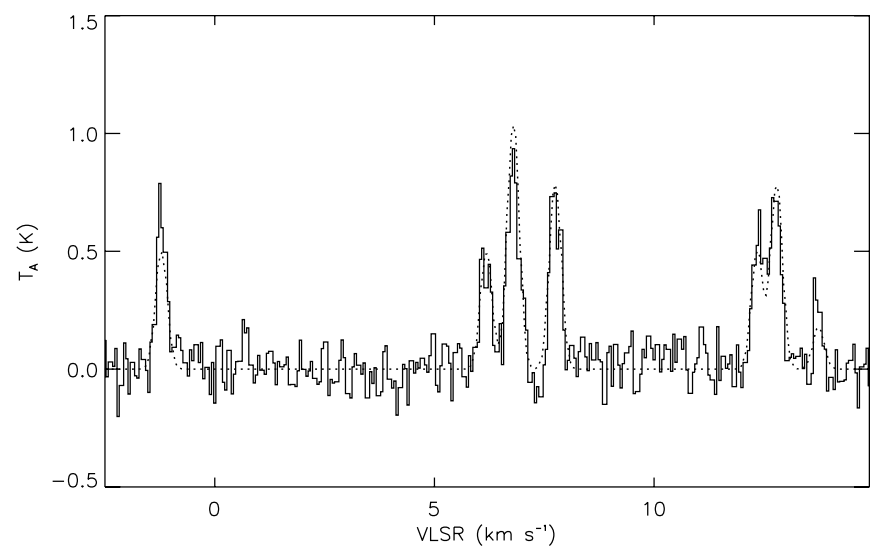

FIG. 10.-First panel of Fig. 6 (top) $\left(\mathrm{N}_{2} \mathrm{H}^{+}[1-0]\right.$; central position $)$ in detail.
The core density from our best-fit model, $n_{\mathrm{H}_{2}}=5 \times$ $10^{5} \mathrm{~cm}^{-3}$, is below that derived from millimeter and submillimeter dust emission observations, $10^{6} \mathrm{~cm}^{-3}$ (Ward-Thompson et al. 1999; Evans et al. 2001; Tafalla et al. 2002), but comparable to the value derived from dust absorption, $4 \times 10^{5} \mathrm{~cm}^{-3}$ (Bacmann et al. 2000). The gas densities derived from the dust emission observations assumed a dust mass opacity of $\kappa_{1.3 \mathrm{~mm}}=0.005 \mathrm{~cm}^{-2} \mathrm{~g}^{-1}$. If one assumes a higher opacity of $\kappa_{1.3 \mathrm{~mm}}=0.04 \mathrm{~cm}^{-2} \mathrm{~g}^{-1}$, a factor of 2 above the millimeter opacity, as suggested by Kruegel \& Siebenmorgen (1994), but still a factor of 25 below that proposed by Ossenkopf \& Henning (1994), then the densities derived from the dust emission observations would be in agreement with the densities derived from both the dust absorption and the molecular line observations.

Our derived abundance, $X\left(\mathrm{~N}_{2} \mathrm{H}^{+}\right) \sim 10^{-9}$, is in agreement with Caselli et al. (2002c) but is higher than that proposed by Tafalla et al. (2002), $X\left(\mathrm{~N}_{2} \mathrm{H}^{+}\right) \sim 8 \times 10^{-11}$. The result of

TABLE 2

Model Parameters for L1489-NH3

\begin{tabular}{|c|c|c|c|}
\hline Parameter & Best Fit & Minimum & Maximum \\
\hline 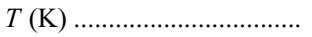 & 14 & 10 & 17 \\
\hline $\log n_{\text {cor }}\left(\mathrm{cm}^{-3}\right) \ldots \ldots \ldots \ldots \ldots \ldots$ & 4.9 & 4.5 & 5.2 \\
\hline$r_{\text {cor }}(\mathrm{pc})$ & 0.015 & 0.006 & 0.020 \\
\hline 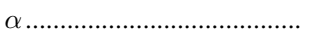 & -1.5 & -2.0 & -1.25 \\
\hline $\log X_{\mathrm{N}_{2} \mathrm{H}^{+}} \ldots \ldots \ldots \ldots \ldots \ldots \ldots \ldots \ldots \ldots$ & -10.0 & -10.4 & -9.5 \\
\hline$v(r)^{a}\left(\mathrm{~km} \mathrm{~s}^{-1}\right) \ldots \ldots \ldots \ldots \ldots \ldots \ldots$ & 0.0 & $\ldots$ & $\ldots$ \\
\hline$\Delta v_{\text {turb }}^{b}\left(\mathrm{~km} \mathrm{~s}^{-1}\right) \ldots \ldots \ldots \ldots \ldots \ldots$ & 0.15 & 0.05 & 0.20 \\
\hline
\end{tabular}



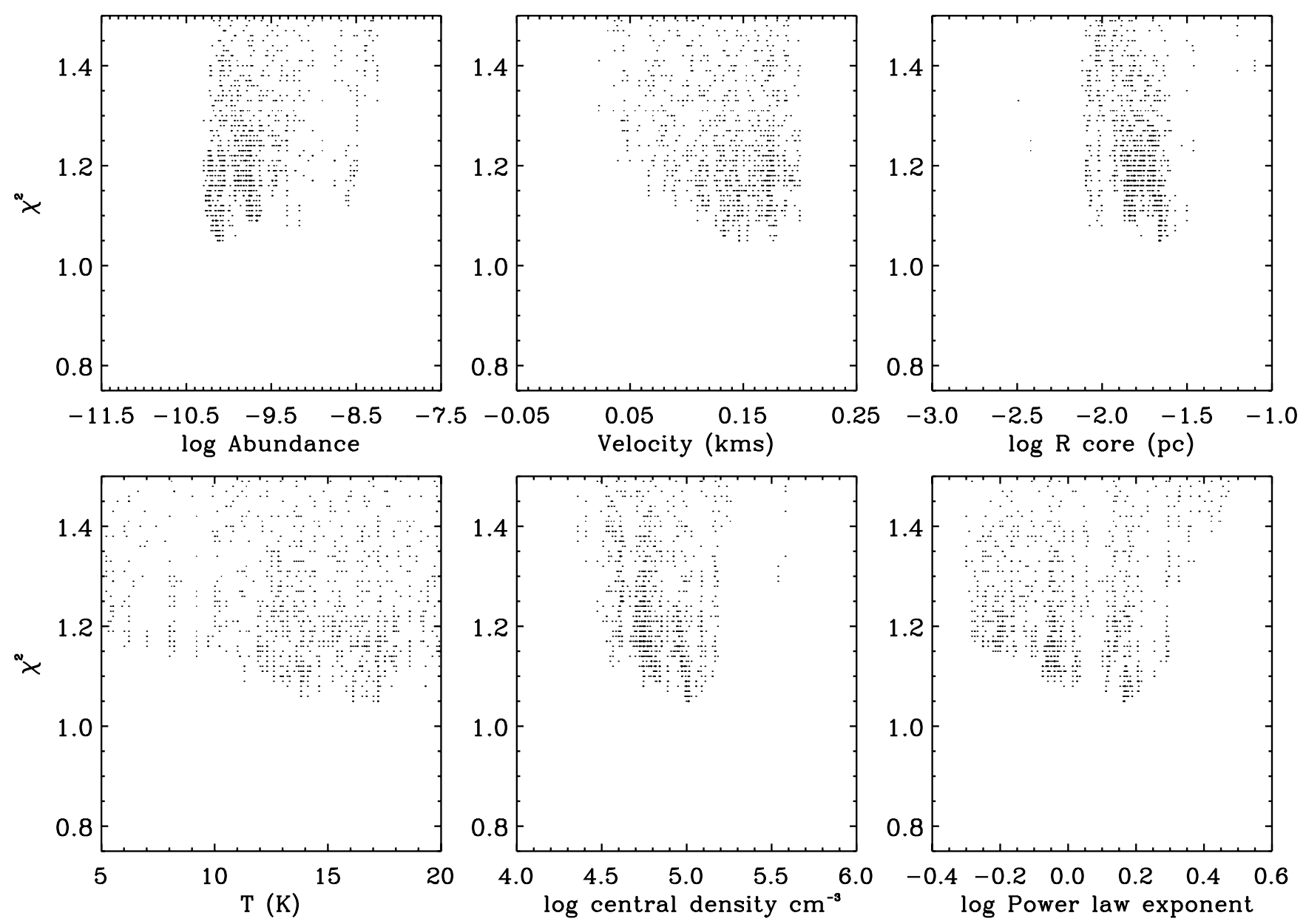

FIG. 11.-Projection of the $\chi^{2}$ surface on the axes of the model parameters for L1489-NH3, in the same format as Fig. 5 .

Tafalla et al. (2002) is based on their $\mathrm{H}_{2}$ densities derived from dust emission, and their lower abundance reflects the higher density given by the dust. In contrast, we use our observations of the $\mathrm{N}_{2} \mathrm{H}^{+}(3-2)$ line and combine these with the same $\mathrm{N}_{2} \mathrm{H}^{+}(1-0)$ observations of Tafalla et al. (2002) to derive both the density and abundance directly from the molecular data alone. Our $J=3-2$ data do not allow the high densities as found from millimeter continuum emission, and to match the intensity of $J=1-0$ emission requires a higher abundance.

Our core radius (called $R_{\text {flat }}$ in Ward-Thompson et al. [1999]; Bacmann et al. 2000) in our best-fit model is $1000 \mathrm{AU}$ and, considering our angular resolution $\left(30^{\prime \prime}=4500 \mathrm{AU}\right.$ at $150 \mathrm{pc}$ ), is comparable to the $1900 \mathrm{AU}$ deduced by Bacmann et al. (2000).

Our best-fit density power law is consistent with the envelope power laws derived from the dust observations of WardThompson et al. (1999) and Bacmann et al. (2000). Evans et al. (2001) suggested that the dust continuum emission, when modeled with a temperature structure decreasing from edge to center, might also be fitted by a density power law consistent with the singular isothermal sphere. We find that our data do not support this hypothesis. As a further check, we also ran some models with the temperature fixed at $5 \mathrm{~K}$ within the core radius and allowing a different temperature as a fitted parameter within the power-law envelope. We also searched models with the abundance described by a power law that decreases with radius $\left(X=X_{0} r^{-m}\right)$. In both cases the fits to the data did not improve, and the estimated densities were not appreciably higher. Thus, in our analysis we find good agreement with the suggestion that the density profile in the prestellar cores flattens toward the center.

One way to understand the constraint against high densities even in the models with lower temperature is to suppose that the emissions were optically thin, the populations were in LTE, and the Rayleigh-Jeans approximation was applicable. Then, to first order the line brightness would be equal to the product of the optical depth and the source function, which is itself proportional to temperature. In this case a change in the temperature by a factor of 2 or 3 would allow only a proportional change in the density if the line brightness were to remain the same. This would be insignificant in terms of a steep $\left(r^{-2}\right)$ power law.

\section{2. $21489-N H 3$}

\subsubsection{Best-Fit Model}

The model for L1489-NH3 is similar to L1544. However, because the observed spectra in L1489-NH3 are consistent with Gaussian profiles and show no evidence for emission self-reversals indicative of infall as seen in L1544, we used a model for L1489-NH3 with a static velocity field, $v(r) \equiv 0$, and included a microturbulent width as a parameter. The spectra of the best-fit model are shown in Figures 9 and 10, the parameter values are given in Table 2 , and the projected $\chi^{2}$ plots are given in Figures 11 and 12. 

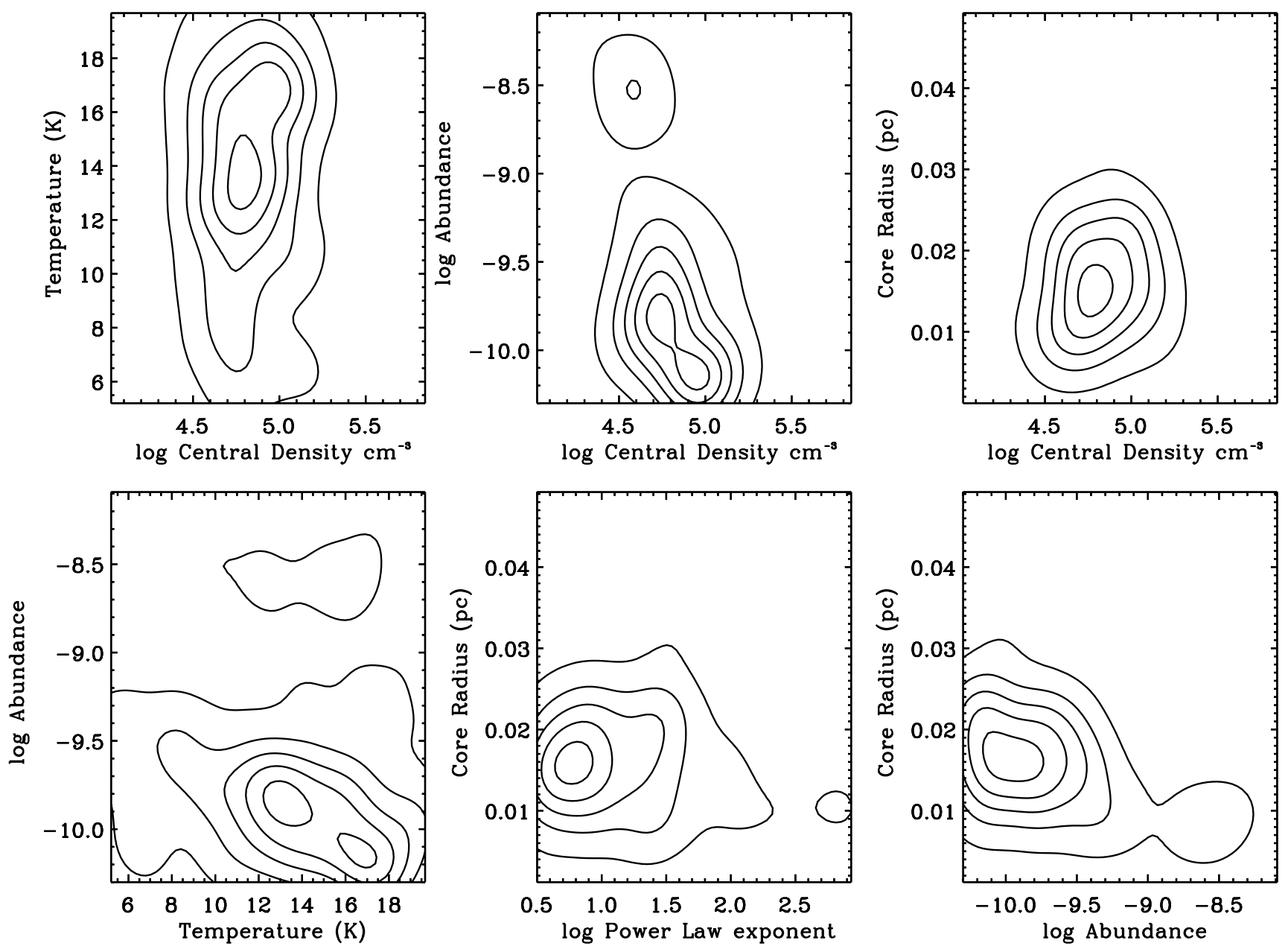

FIG. 12. $-\chi^{2}$ surface for the L1489-NH3 models as a function of some of the model parameters taken two at a time, in the same format as Fig. 8. The contour values of $\chi^{2}$ are $1.1-1.5$ in steps of 0.1 .

The lower values of $\chi^{2}$ in the L1489-NH3 models compared with L1544 are a result of the lower signal-to-noise ratios of the L1489-NH3 spectra compared with that of L1544. The reason why variations of $\chi^{2}$ of less than unity are still significant can be understood by examination of the leastsquares-fitting process. In the modeled and observed spectra, a signal is expected only in certain channels at specific frequencies depending on the hyperfine structure, velocity field, and line width. Channels with no signal may be considered significant or not, depending on where emission is expected. However, it is not possible to know or specify with certainty which channels in the model and data are significant in order to include only the significant channels in the computation of $\chi^{2}$. Channels that have no significant line emission but only noise will contribute an average value of unity to the $\chi^{2}$. In noisy spectra (such as for L1489-NH3 and L1517B) the relatively larger number of channels that are free of emission above the noise produces values of $\chi^{2}$ that are all closer to unity and have a smaller variation between the models. Nonetheless, the relative variations in $\chi^{2}$ are still significant, and, for example, models with $\chi^{2}$ of 1.5 are noticeably much worse than the best-fit model with a $\chi^{2}$ of 1.2 .

Figure 12 explores the possible correlations among the parameters. Here because the data have a lower signal-to-noise ratio and the ratio of the cloud size to angular resolution is lower, our analysis is not as successful in uniquely character- izing the individual parameters. The plots show correlations between the abundance and each of the three parameters, the temperature, density, and core size. Still, the ranges of the fitted parameters, as seen in either the plots of the projected $\chi^{2}$ surface or the contoured surfaces, are narrow enough to be interesting relative to our current knowledge of these clouds. We expect that better data would result in more tightly constrained parameters.

\subsubsection{Comparison with Other Results}

For L1489-NH3 there is no previous analysis with regards to the density structure from dust emission or absorption in the literature. The cloud was mapped in dust emission by Motte \& Andre (2001), who estimated a total flux of $55 \mathrm{mJy}$ beam $^{-1}$ toward the peak. Using this flux, we estimate the average density within the $13^{\prime \prime}$ beam as $10^{6} \mathrm{~cm}^{-3}$ (assuming $\kappa_{1.3 \mathrm{~mm}}=$ $0.005 \mathrm{~cm}^{2} \mathrm{~g}^{-1}$ and a dust temperature of $10 \mathrm{~K}$ ). Assuming that this average value is a lower limit to the density in the core of a core-envelope structure, we find that, similar to L1544, the density, $n_{\text {cor }}=10^{5} \mathrm{~cm}^{-3}$, derived from $\mathrm{N}_{2} \mathrm{H}^{+}$is below that estimated via dust emission. When we use the higher dust mass opacity of $\kappa_{1.3 \mathrm{~mm}}=0.04 \mathrm{~cm}^{2} \mathrm{~g}^{-1}$, the gas densities estimated from the dust and molecular line observations are consistent. Our estimated temperature of $17 \mathrm{~K}$ is higher than, but, as indicated by the $\chi^{2}$ distribution in Figure 11, consistent with, the $9.5 \mathrm{~K}$ reported by Jijina et al. (1999) from observations of $\mathrm{NH}_{3}$. 

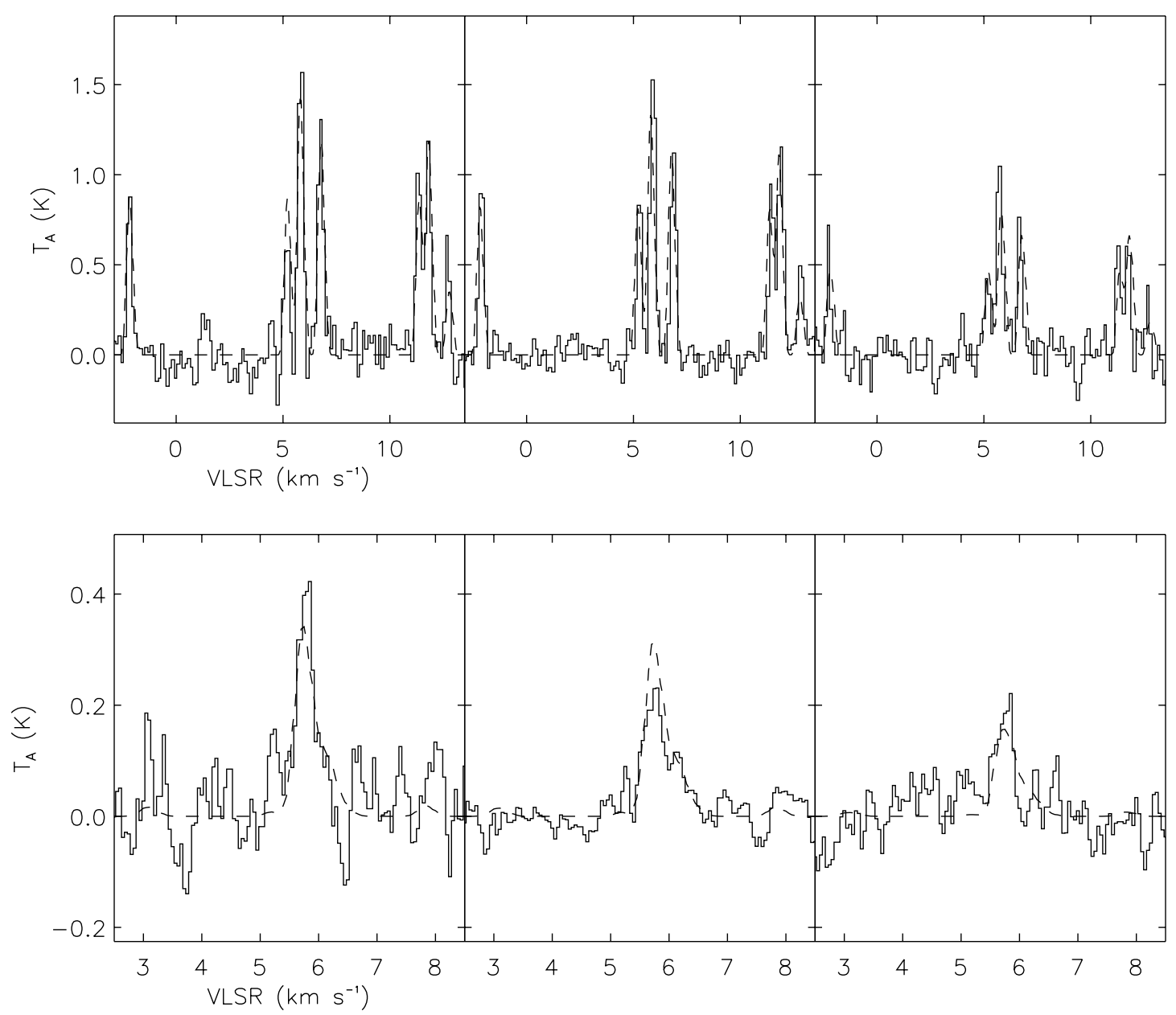

Fig. 13.- Spectra (solid line) and model (dashed line) for L1517B, in the same format as Fig. 3.

\section{3. $L 1517 B$}

\subsubsection{Best-Fit Model}

For this cloud we have adopted the same model as described for L1489-NH3. The spectra are similar to L1489-NH3 in that they have Gaussian profiles broadened only slightly above thermal. As in L1489-NH3, the data do not discriminate between a wide variety of models with different weak velocity fields. The best-fit model is shown in Figures 13 and 14, the model parameters are summarized in Table 3 , and the $\chi^{2}$

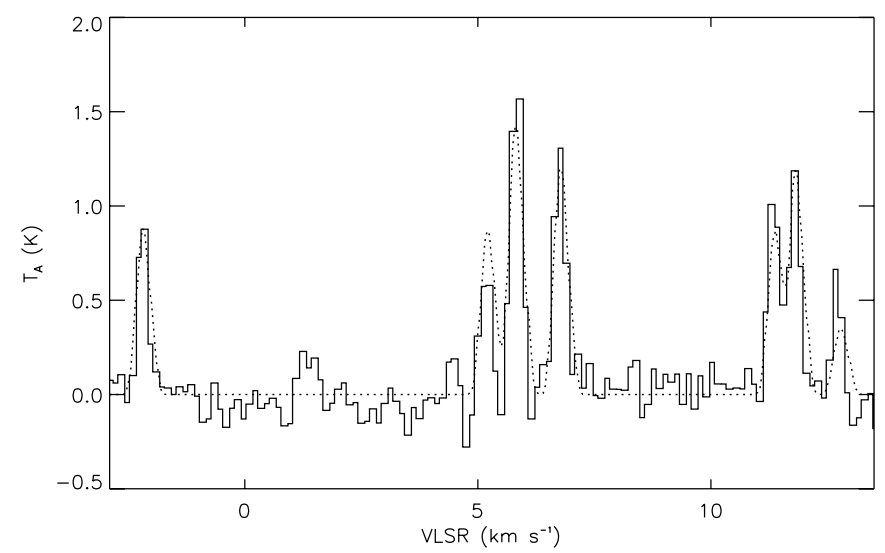

FIG. 14.-First panel of Fig. $13\left(\mathrm{~N}_{2} \mathrm{H}^{+}[1-0]\right.$; central position $)$ in detail. distributions are given in Figures 15 and 16. There are correlations between the abundance and the temperature and core size similar to those seen in the analysis of L1489-NH3.

\subsubsection{Comparison with Other Results}

Tafalla et al. (2002) included L1517B in their study, finding a central density of $\sim 2 \times 10^{5} \mathrm{~cm}^{-3}$ from dust emission and an $\mathrm{N}_{2} \mathrm{H}^{+}$abundance of $\sim 10^{-10}$ from $\mathrm{N}_{2} \mathrm{H}^{+} J=1-0$ observations. While this gas density derived from the dust emission assumes the lower dust mass opacity of $\kappa_{1.3 \mathrm{~mm}}=0.005 \mathrm{~cm}^{2} \mathrm{~g}^{-1}$ and is consistent with the core gas density that we derive from $\mathrm{N}_{2} \mathrm{H}^{+}$emission, the gas column density derived from the dust is almost an order of magnitude higher than the gas column density derived from the molecular line observations. This is because the radius of the high-density core derived from the

TABLE 3

Model Parameters for L1517B

\begin{tabular}{|c|c|c|c|}
\hline Parameter & Best Fit & Minimum & Maximum \\
\hline$T(\mathrm{~K})$ & 9 & 7 & 15 \\
\hline $\log n_{\text {cor }}\left(\mathrm{cm}^{-3}\right)$ & 5.1 & 4.8 & 5.2 \\
\hline$r_{\mathrm{cor}}(\mathrm{pc})$ & 0.022 & 0.006 & 0.030 \\
\hline$\alpha$ & -1.4 & -2.0 & -0.8 \\
\hline $\log X_{\mathrm{N}_{2} \mathrm{H}^{+}} \ldots \ldots \ldots \ldots \ldots \ldots \ldots$ & -9.8 & -10.1 & -8.0 \\
\hline$v(r)^{a}\left(\mathrm{~km} \mathrm{~s}^{-1}\right) \ldots \ldots \ldots \ldots \ldots$ & 0.0 & $\ldots$ & 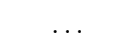 \\
\hline$\Delta v_{\text {turb }}^{b}\left(\mathrm{~km} \mathrm{~s}^{-1}\right) \ldots \ldots \ldots \ldots \ldots$ & 0.17 & 0.12 & 0.2 \\
\hline
\end{tabular}



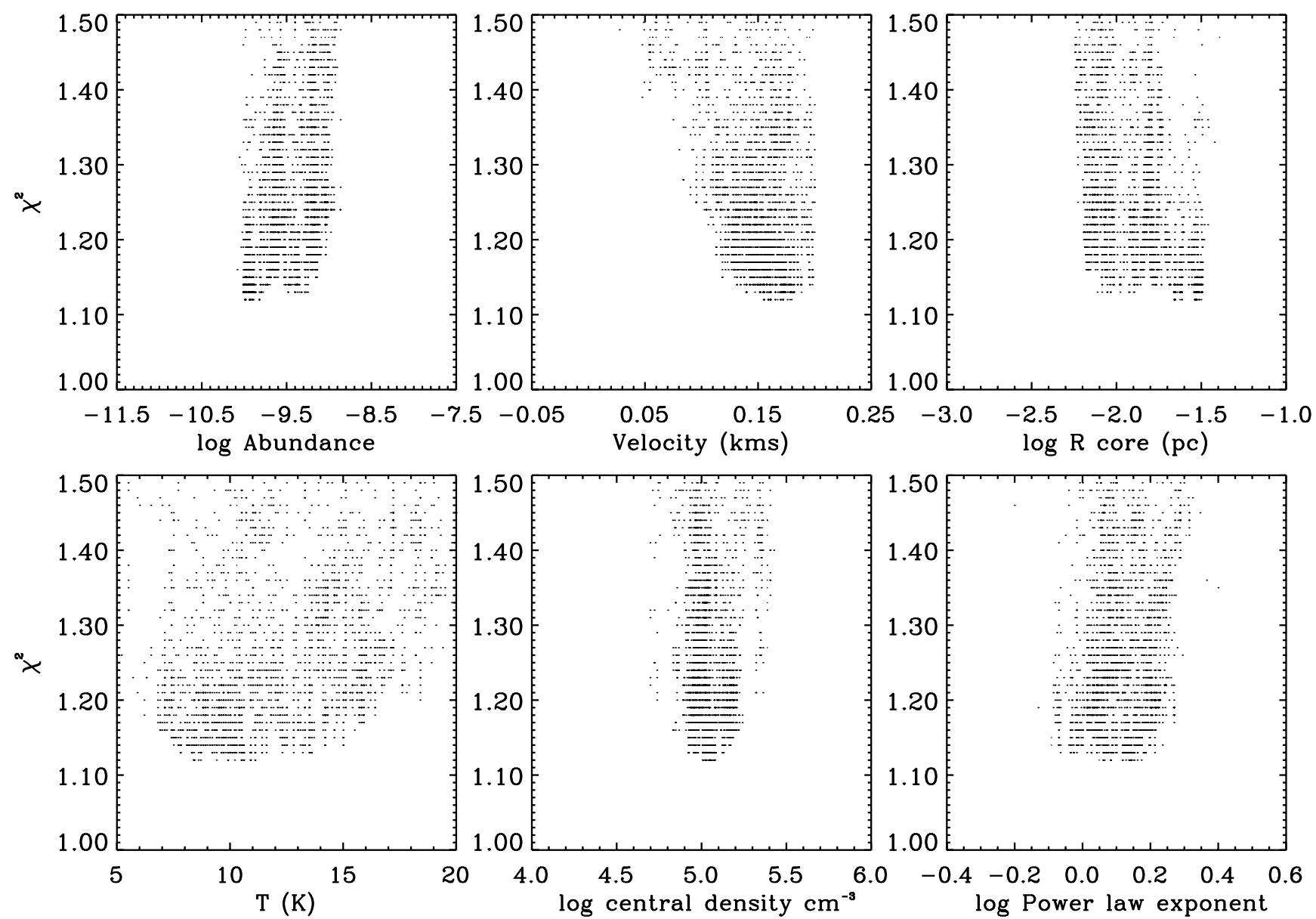

FIG. 15.-Projection of the $\chi^{2}$ surface on the axes of the model parameters for L1517B, in the same format as Fig. 5.

dust emission (Tafalla et al. 2004) is a factor of 8 larger than the core radius that we derive from our $\mathrm{N}_{2} \mathrm{H}^{+}$observations. Thus, the molecular line observations again indicate a higher dust mass opacity closer to $\kappa_{1.3 \mathrm{~mm}}=0.04 \mathrm{~cm}^{2} \mathrm{~g}^{-1}$. Because we have modeled spectra at only three positions in L1517B that are along a strip just off the center of the cloud, our estimate of the size of the core is not well constrained by our data.

\section{DISCUSSION}

In two of the cores in this study, L1489-NH3 and the wellstudied core L1544, we find that the density of molecular hydrogen as determined by a gas tracer $\left(\mathrm{N}_{2} \mathrm{H}^{+}\right)$is about an order of magnitude below that previously derived from dust observations if the dust mass opacity is assumed to be $\kappa_{1.3 \mathrm{~mm}}=0.005 \mathrm{~cm}^{2} \mathrm{~g}^{-1}$. In the third cloud in our study, L1517B, the estimates of the gas column density based on dust and molecular emission are similarly discrepant. This discrepancy has also been found in other studies. Chini et al. (1993) and Kruegel \& Chini (1994) computed the mass of the HH 24 MMS core via dust $(1.3 \mathrm{~mm}$ emission $)$ and gas $\left(\mathrm{C}^{18} \mathrm{O}\right)$ and found a 2 orders-of-magnitude discrepancy (with the core mass derived from $\mathrm{C}^{18} \mathrm{O}$ below that estimated from dust). They argue that freezeout of $\mathrm{C}^{18} \mathrm{O}$ could not account for all this difference.

Different models of the structure of interstellar dust yield different estimates of the dust mass opacity. The opacity of $\kappa_{1.3 \mathrm{~mm}}=0.005 \mathrm{~cm}^{2} \mathrm{~g}^{-1}$ is based on a model of grains in which most of the opacity in the millimeter wavelengths is due to silicates (Pollack et al. 1994). This model might be more appropriate for dust in a warmer environment with radiative energy heating the dust. Kruegel \& Siebenmorgen (1994) considered a dust model for colder environments that includes the effect of coagulation of dust grains into fluffy aggregates and the formation of ice mantles around the dust. They calculate a higher dust mass opacity $\kappa_{1.3 \mathrm{~mm}}=0.02 \mathrm{~cm}^{2} \mathrm{~g}^{-1}$, which would yield gas densities from dust that are more consistent with the molecular line observations. Ossenkopf \& Henning (1994) derived a higher opacity, $\kappa_{1.3 \mathrm{~mm}}=1.0 \mathrm{~cm}^{2}$ $\mathrm{g}^{-1}$, for dust composed of fluffy structures and ice mantles.

Our observations suggest that the dust mass opacity in the starless cores may be close to $\kappa_{1.3 \mathrm{~mm}}=0.04 \mathrm{~cm}^{2} \mathrm{~g}^{-1}$, consistent with grain models with enhanced opacities due to fluffy aggregates and ice mantles that might be found in the extremely cold, dark interiors of the starless cores.

One way to understand the constraint against higher gas densities imposed by the molecular line observations is to examine the simple "critical density" argument for radiative excitation. Similar to our discussion of correlated parameters in $\S 2.1$, this argument is intended to illustrate the analysis performed by the numerical RT modeling rather than to be an argument in itself. All the quantitative estimates derived in this study are made using the full numerical code and not by order-of-magnitude arguments.

The observed ratio of the (3-2) and $(1-0) \mathrm{N}_{2} \mathrm{H}^{+}$lines suggests a gas density sufficiently low that the lines are not thermalized, and therefore, the density must be below the critical density for collisional excitation. For example, the observed corrected antenna temperatures of the $\mathrm{N}_{2} \mathrm{H}^{+}(1-0)$ and $\mathrm{N}_{2} \mathrm{H}^{+}$ 

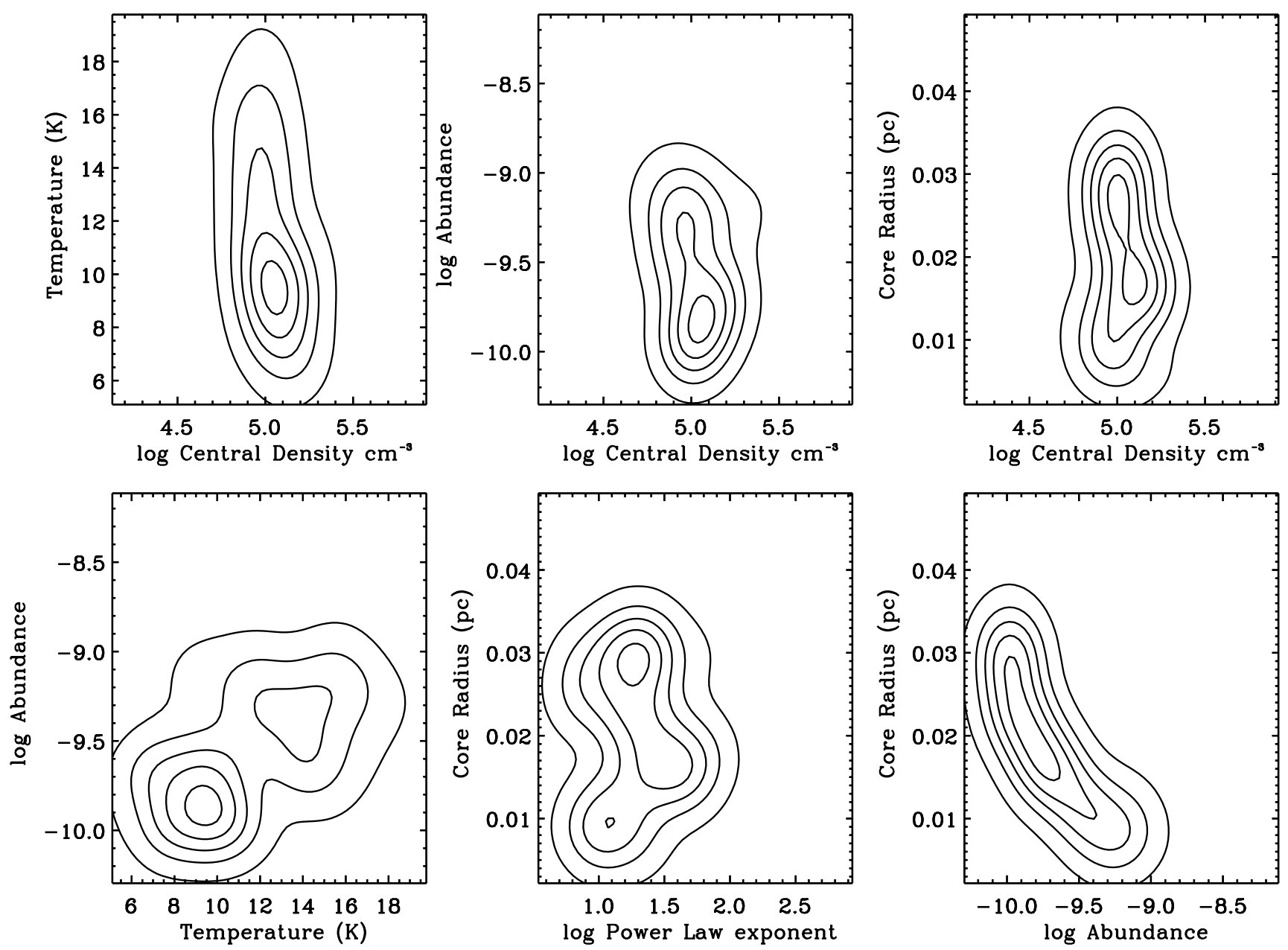

Fig. 16. - $\chi^{2}$ surface for the L1517B models as a function of some of the model parameters taken two at a time, in the same format as Fig. 8. The contour values of $\chi^{2}$ are $1.1-1.5$ in steps of 0.2 .

(3-2) main hyperfine lines in the center position of L1544 are 4.1 and $1.0 \mathrm{~K}$, respectively. Using the brightnesses of the satellite hyperfine lines in each of the two transitions implies optical depths of roughly 1.7 for the (1-0) main hyperfine and 2.7 for the (3-2) main hyperfine. If both the (1-0) and (3-2) lines were thermalized at a temperature of $8.5 \mathrm{~K}$, the brightnesses would be about 4.3 and $3.2 \mathrm{~K}$, the latter significantly higher than the observed (3-2) brightness of $1 \mathrm{~K}$.

The weakness of the (3-2) line implies a low excitation temperature, significantly below $8.5 \mathrm{~K}$. However, the gas temperature cannot be much lower than $8.5 \mathrm{~K}$, because if it were, the observed $4.1 \mathrm{~K}$ brightness of the $(1-0)$ line would require superthermal excitation. The low (3-2) excitation temperature then implies that the density must be low enough that radiative de-excitation of the (3-2) line is significant compared with collisional de-excitation. The density must be such that the collision rate, $n\left(\mathrm{H}_{2}\right) q_{32}$, is less than $A_{32}$, but $n\left(\mathrm{H}_{2}\right) q_{10}$ is greater than $A_{10}$. The Einstein $A$-coefficients for the $(1-0)$ and $(3-2)$ lines are $A_{10}=3.62 \times 10^{-5}$ and $A_{32}=1.26 \times 10^{-3} \mathrm{~s}^{-1}$. The collisional rate coefficients for $\mathrm{N}_{2} \mathrm{H}^{+}$should be approximately the same as those of the similar molecular ion $\mathrm{HCO}^{+}$, which at a gas temperature of $10 \mathrm{~K}$ are $q_{10}=2.6 \times 10^{-10}$ and $q_{32}=$ $4.5 \times 10^{-10} \mathrm{~cm}^{3} \mathrm{~s}^{-1}$ (Flower 1999; Table 1). Thus, to an order of magnitude, the gas density in the starless cores should be between $10^{5}$ and $10^{6} \mathrm{~cm}^{-3}$ within most of the volume of the cloud.
Possibilities other than a higher dust mass opacity that would reconcile the difference between the dust and molecular line estimates cannot be ruled out. The collision rates estimated for $\mathrm{N}_{2} \mathrm{H}^{+}$with $\mathrm{H}_{2}$ may be lower than adopted in our calculations, or, alternatively, the $\mathrm{N}_{2} \mathrm{H}^{+}$may be depleted in the centers of the cores. Our models preclude a temperature gradient to account for this difference since a temperature gradient would only enhance the discrepancy (Evans et al. 2001).

In our calculations we have adopted the collision rates for $\mathrm{HCO}^{+}$for use with $\mathrm{N}_{2} \mathrm{H}^{+}$. (Flower 1999). Given the similarity in molecular structure, $\mathrm{HCO}^{+}$is believed to be a reasonable substitute for $\mathrm{N}_{2} \mathrm{H}^{+}$(Monteiro 1984). If the collision process is less efficient than theoretical estimates, then higher densities would be required to match the observed line ratios. Experimental measurements of the $\mathrm{HCO}^{+}-\mathrm{H}_{2}$ collision rates have been performed by Oesterling et al. (2001), who noted that their experimental values tended to lie below the theoretical numbers, but by less than a factor of 2 . However, the experiment included both ortho and para forms of $\mathrm{H}_{2}$, so a direct comparison with theory, which does not include ortho- $\mathrm{H}_{2}$ as a collision partner, was not feasible.

Current theory suggests that although molecular ions do not freeze directly onto grain surfaces, depletion of ionic species from the gas phase may occur if the parent molecule $\left(\mathrm{N}_{2}\right.$ for $\mathrm{N}_{2} \mathrm{H}^{+}$) is frozen onto the surfaces of the dust grains (Bergin et al. 2002). If $\mathrm{N}_{2} \mathrm{H}^{+}$were absent in the densest regions of 
these cores, then the densities could be arbitrarily high without any effect on the observed $\mathrm{N}_{2} \mathrm{H}^{+}$emission. In our analysis we have examined models in which the $\mathrm{N}_{2} \mathrm{H}^{+}$abundance was allowed to decrease in the central regions. Some of these models were able to achieve reasonable fits to the data, but we did not find solutions that improved the fit. Therefore, we have considered for discussion only the simpler models with constant abundance, but our analysis does not rule out a variable abundance of $\mathrm{N}_{2} \mathrm{H}^{+}$.

\section{CONCLUSIONS}

1. We have developed a method of analysis to derive the gas temperature, density, velocity, and molecular abundance from radio-frequency spectral line data by means of an RT model and a heuristic search algorithm based on SA. The RT model includes a new technique to incorporate the hyperfine components into the RT that allows acceleration of the $\Lambda$-iteration with overlapping hyperfine lines. This technique provides an approximate treatment for molecules whose collision rates between individual hyperfine levels are not known. We also demonstrate a means to test the accuracy of an RT code against a simple semianalytic calculation.

2. We show how the results of the search for a best-fit model can be used to estimate the uncertainties in the derived physical data, the temperature, density, velocity, and abundance. We find that with good enough observational data, that is to say, sufficiently high signal-to-noise ratio and angular and spectral resolution, the uncertainties in the derived results are not strongly correlated. Rather, temperature, density, and abundance can be estimated independently.

3. As an example, we apply our method of analysis to new observations of $\mathrm{N}_{2} \mathrm{H}^{+}$toward a sample of starless cores, L1544, L1489-NH3, and L1517B.
4. The observed line ratios require that the gas in the cores be sufficiently rarefied that the $\mathrm{N}_{2} \mathrm{H}^{+}$lines are not thermalized. In particular, the $\mathrm{H}_{2}$ density within the cores must be less than $\sim 3 \times 10^{6} \mathrm{~cm}^{-3}$. Thus, the density gradient cannot increase inward to small radii as a power law with any significant exponent. We find that models with constant density at small radii are consistent with the data.

5. The densities derived from the molecular line emission are lower than those derived from dust emission if the dust mass opacity is $\kappa_{1.3 \mathrm{~mm}}=0.005 \mathrm{~cm}^{2} \mathrm{~g}^{-1}$, a value suitable for silicate grains. A dust mass opacity of $\kappa_{1.3 \mathrm{~mm}}=0.04 \mathrm{~cm}^{2} \mathrm{~g}^{-1}$, suggesting grain models as fluffy aggregates with ice mantles, yields gas densities from the dust observations that are consistent with the molecular line observations.

6. With the limited data, we are not able to determine whether the temperature in the starless cores decreases inwardly or is constant. It is possible to construct equally good models with constant as well as with lower central temperatures. Observations at higher angular resolution or with additional spectral lines would be required to discriminate between these models.

7. The observed velocity fields in L1489-NH3 and L1517B are consistent with gravitational free fall, a static field with microturbulence, or other weak velocities. The $\mathrm{N}_{2} \mathrm{H}^{+}$(1-0) spectrum from the central position of L1544 shows strong asymmetry that cannot be fitted by turbulence alone, implying some inward motion. The best agreement with the data is provided by infall that accelerates inward, but the maximum velocity at the cloud center can be no larger than a few times the thermal velocity.

The authors thank Paola Caselli and Mario Tafalla for the use of their data and Luca Dore for the calculations of the $\mathrm{N}_{2} \mathrm{H}^{+}$hyperfine structure.

\section{APPENDIX}

\section{COMPARISON SOLUTIONS FOR SPHERICAL GEOMETRY}

For purposes of testing three-dimensional numerical RT codes, it is useful to have accurate analytic or semianalytic solutions for comparison. Such solutions can easily give high accuracy for comparison with numerical codes.

In this appendix we compare our numerical code with the "standard" two-level atom problem in an isothermal constant-density sphere. This spherical problem can be reduced to an equivalent plane-parallel problem by a method that originated in the neutron transport literature (see, e.g., Davison 1957, p. 96). This method was applied to line transfer in a two-level atom by Sobolev (1962) and Cuperman et al. $(1963,1964)$. The latter authors solved the resulting integral equation using discrete ordinates in angle and frequency. Here we use the kernel approximation method as given by Kunasz \& Hummer (1974).

For plane-parallel media the standard two-level atom problem (see, e.g., Avrett \& Hummer 1965) can be formulated in terms of an integral equation with kernel function

$$
K(\tau)=\frac{1}{2} \int_{-\infty}^{\infty} \phi_{x}^{2} E_{1}\left(\phi_{x} \tau\right) d x=\frac{1}{2} \int_{-\infty}^{\infty} d x \phi_{x}^{2} \int_{0}^{1} \frac{d \mu}{\mu} e^{-\phi_{x} \tau / \mu}
$$

which is normalized to unity,

$$
\int_{-\infty}^{\infty} K(|\tau|) d \tau=1
$$

The kernel approximation method in plane-parallel geometry (Avrett \& Hummer 1965) involves replacing the kernel function $K(\tau)$ by an approximation as a sum of $N$ exponentials:

$$
K(\tau)=\sum_{i=1}^{N} a_{i} e^{-b_{i} \tau}
$$



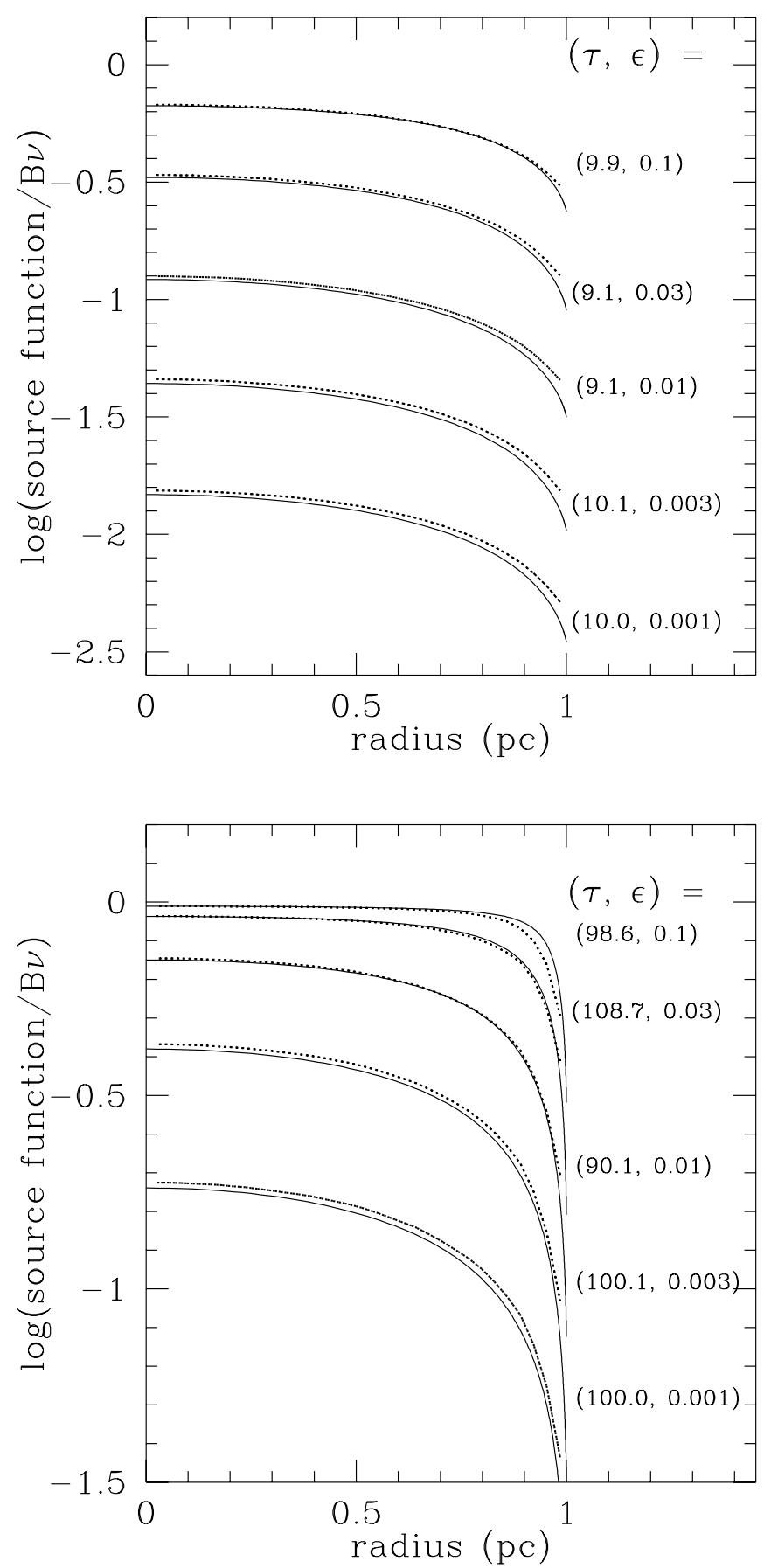

Fig. 17.-Source functions vs. optical depth for various values of radial optical depth $\tau$ and $\epsilon$. Plotted are results using the accurate semianalytic method (solid line) and the numerical code (dotted line).

The normalization of the kernel, as given in equation (A2), implies that the coefficients of the kernel approximation should satisfy

$$
\sum_{i} \frac{a_{i}}{b_{i}}=\frac{1}{2}
$$

The constants $a_{i}$ and $b_{i}$ can be determined by introducing quadrature rules over $x$ and $\mu$ in the integral (eq. [A1]). Alternatively, they can be determined by fitting exponentials to accurate numerical values of the kernel, as was done by Avrett \& Hummer (1965). This is especially useful when the required frequency bandwidth becomes large, as it does for Voigt profiles with sufficiently large optical depths. In such cases kernel fits should achieve a given accuracy in many fewer terms than the discrete ordinate method. Extensive kernel fits are given in Avrett \& Loeser (1966).

We now consider an isothermal constant-density spherical medium of radius $R$ in which the absorption coefficient is spatially constant. The mean optical depth from the center to the surface is denoted by $T$ (the line center optical thickness $T_{0}$ is a factor of $1 / \sqrt{\pi}$ times this). We employ the usual notations $B$ for the Planck function in the line and $\epsilon$ for the ratio of $C_{21} /\left(A_{21}+C_{21}\right)$, where $C_{21}$ is the collisional rate coefficient and $A_{12}$ is the Einstein $A$-coefficient for a transition from the upper to the lower levels. 
As shown by Kunasz \& Hummer (1974), the solution for this standard two-level problem in a sphere can be written

$$
S(\tau)=B\left[1-\sum_{j=1}^{N} L_{j} \frac{2 \sinh \left(k_{j} \tau\right)}{\tau}\right] .
$$

The $L_{j}$ and $k_{j}$ are constants determined by the equations

$$
\begin{gathered}
1=(1-\epsilon) \sum_{i} \frac{2 a_{i} b_{i}}{b_{i}^{2}-k_{j}^{2}}, \\
\frac{T}{b_{i}}+\frac{1}{b_{i}^{2}}=\sum_{j}\left(\frac{e^{k_{j} T}}{b_{i}-k_{j}}-\frac{e^{-k_{j} T}}{b_{i}+k_{j}}\right) L_{j} .
\end{gathered}
$$

The first condition (A6) is the characteristic equation for the unknown constants $k_{j}$. This is equivalent to an $n$ th-order polynomial equation in $k_{j}^{2}$, which determines $n$ positive values of $k_{j}$. More practically, the values of $k_{j}^{2}$ interleave the values of $b_{i}^{2}$ and may be easily found by Newton's method.

The last condition (A7) is then a linear system of equations for the unknown constants $L_{j}$, which can easily be solved numerically. The source function is then given by equation (A5).

To check our three-dimensional RT code, we considered a set of constant-property spherical models for a variety of values of $\tau$ and $\epsilon$. The solid curves in Figure 17 are accurate results for the source functions using the above semianalytic method. We then numerically solved the spherical problem on a uniform cubical grid, which gave solutions that were approximately spherically symmetric, as expected. The numerical results shown as the dotted curves in Figure 17 are for a radial ray parallel to the grid. One sees that the accuracy of the numerical results is quite good except near the sharp outer boundary of the sphere, where the true solution varies over a scale too small to be resolved by our discretization. We expect better accuracy for cases in which the density decreases outwardly more smoothly.

\section{REFERENCES}

Aikawa, Y., Ohashi, N., Inutsuka, S., Herbst, E., \& Takakuwa, S. 2001, ApJ, 552,639

Alves, J., Lada, C., \& Lada, E. 2001, Nature, 409, 159

Andre, P., Ward-Thompson, D, \& Motte, F. 1996, A\&A, 314, 625

Avrett, E. H., \& Hummer, D. G. 1965, MNRAS, 130, 295

Avrett, E. H., \& Loeser, R. 1966 (SAO SP-201; Cambridge: SAO)

Bacmann, A., Andre, A. P., Puget, J.-L., Abergel, A., Bontemps, S., \& WardThompson, D. 2000, A\&A, 361, 555

Beichman, C. A., Myers, P. C., Emerson, J. P., Harris, S., Mathieu, R., Benson, P. J., \& Jennings, R. E. 1986, ApJ, 307, 337

Benson, P., \& Myers, P. 1989, ApJS, 71, 89

Bergin, E., Alves, J., Huard, T., \& Lada, C. 2002, ApJ, 570, L101

Bergin, E., Langer, W., \& Goldsmith, P. 1995, ApJ, 441, 222

Bonnor, W. 1956, MNRAS, 116, 351

Brown, P., Charnley, S., \& Millar, T. 1988, MNRAS, 231, 409

Caselli, P., Benson, P., Myers, P., \& Tafalla, M. 2002a, ApJ, 572, 238

Caselli, P., Myers, P., \& Thaddeus, P. 1995, ApJ, 455, L77

Caselli, P., Walmsley, C. M., Tafalla, M., Dore, L., \& Myers, P. C. 1999, ApJ, 523, L165

Caselli, P., Walmsley, C. M., Zucconi, A., Tafalla, M., Dore, L., \& Myers, P. C. 2002b, ApJ, 565, 331

2002c, ApJ, 565, 344

Chini, R., Kruegel, E., Haslam, C. G. T., Kreysa, E., Lemke, R., Reipurth, B., Sievers, A., \& Ward-Thompson, D. 1993, A\&A, 272, L5

Cuperman, S., Engelmann, F., \& Oxenius, J. 1963, Phys. Fluids, 6, 108 1964, Phys. Fluids, 7, 428

Davison, B. 1957, Neutron Transport Theory (Oxford: Clarendon)

Evans, N., Rawlings J., Shirley, Y., \& Mundy, L. 2001, ApJ, 557, 193

Flower, D. R. 1999, MNRAS, 305, 651

Foster, P., \& Chevalier, R. 1993, ApJ, 416, 303

Goncalves, J., Galli, D., \& Walmsley, M. 2004, A\&A, 415, 617

Hasegawa, T. 1988, PASJ, 40, 219

Hasegawa, T., \& Herbst, E. 1993, MNRAS, 261, 83

Hasegawa, T., Herbst, E., \& Leung, C. 1992, ApJS, 82, 167

Hildebrand, R. H., Loewenstein, R. F., Harper, D. A., Orton, G. S., Keene, J., \&

Whitcomb, S. E. 1985, Icarus, 64, 64

Hunter, C. 1977, ApJ, 218, 834

Jijina, J., Adams, F., \& Myers, P. 1999, ApJS, 125, 161

Keto, E. 1990, ApJ, 355, 190

Kruegel, E., \& Chini, R. 1994, A\&A, 287, 947

Kruegel, E., \& Siebenmorgen, R. 1994, A\&A, 288, 929
Kunasz, P., \& Auer, L. H. 1988, J. Quant. Spectrosc. Radiat. Transfer, 39, 67

Kunasz, P., \& Hummer, D. G. 1974, MNRAS, 166, 19

Larson, R. 1969, MNRAS, 145, 271

Larson, R., \& Starrfield, S. 1971, A\&A, 13, 190

Lee, C. W., \& Myers, P. C. 1999, ApJS, 123, 233

Lee, C. W., Myers, P. C., \& Tafalla, M. 2001, ApJS, 136, 703

Metropolis, N., Rosenbluth, A., Rosenbluth, M., Teller, A., \& Teller, E. 1953, J. Chem. Phys., 21, 1087

Monteiro, T. 1984, MNRAS, 210, 1

Motte, F., \& Andre, P. 2001, A\&A, 365, 440

Myers, P., \& Benson, P. 1983, ApJ, 266, 309

Myers, P., Linke, R., \& Benson, P. 1983, ApJ, 264, 517

Oesterling, L. C., de Lucia, F. C., \& Herbst, E. 2001, Spectrochim. Acta A, 57, 705

Ossenkopf, V., \& Henning, Th. 1994, A\&A, 291, 943

Pickett, H. 1991, J. Mol. Spectrosc., 148, 371

Pollack, J., Hollenbach, D., Beckwith, S., Simonelli, D., Rough, T., \& Fong, W. 1994, ApJ, 421, 615

Rybicki, G., \& Hummer, D. 1991, A\&A, 245, 171 1992, A\&A, 262, 209

Shirley, Y. L., Evans, N. J., II, \& Rawlings, J. M. C. 2002, ApJ, 575, 337

Shu, F. 1977, ApJ, 214, 488

Sobolev, V. V. 1962, AZh, 39, 632 (translated in Soviet Astron., 6, 497 [1963])

Stamatellos, D., \& Whitworth, A. 2003, A\&A, 407, 941

Szu, H., \& Hartley, R. 1987, Phys. Lett. A, 122, 157

Tafalla, M., Mardones, D., Myers, P., Caselli, P., Bachiller, R., \& Benson, P. 1998, ApJ, 504, 900

Tafalla, M., Myers, P., Caselli, P., \& Walmsley, M. 2004, A\&A, 416, 191

Tafalla, M., Myers, P., Caseli, P., Walmsley, C., \& Comito, C. 2002, ApJ, 569,815

Townes, C., \& Schawlow, A. 1955, Microwave Spectroscopy (New York: Dover)

Ward-Thompson, D., Motte, F., \& Andre, P. 1999, MNRAS, 305, 143

Ward-Thompson, D., Scott, P. F., Hills, R. E., \& Andre, P. 1994, MNRAS, 268,276

Whitworth, A., \& Summers, D. 1985, MNRAS, 214, 1

Whitworth, A., \& Ward-Thompson, D. 2001, ApJ, 547, 317

Willacy, K., \& Williams, D. 1993, MNRAS, 260, 635

Williams, J. P., Myers, P. C., Wilner, D. J., \& di Francesco, J. 1999, ApJ, 513, L61

Zucconi, A., Walmsley, C., \& Galli, D. 2001, A\&A, 376, 650 